\title{
Molecular Mechanism of Taurocholate Transport by the Bile Salt Export Pump, an ABC Transporter Associated with Intrahepatic Cholestasis $\mathbf{S}$
}

\author{
Muhammad Imran Sohail, Diethart Schmid, Katrin Wlcek, Matthias Spork, Gergely Szakács, \\ Michael Trauner, Thomas Stockner, and Peter Chiba \\ Institute of Medical Chemistry, Center for Pathobiochemistry and Genetics (M.I.S., M.S., P.C.), Institute of Physiology, Center for \\ Physiology and Pharmacology (D.S.), Institute of Cancer Research (G.S.), Hans Popper Laboratory of Molecular Hepatology, \\ Division of Gastroenterology and Hepatology, Department of Internal Medicine III (M.T.), and Institute of Pharmacology, Center \\ for Physiology and Pharmacology (T.S.), Medical University of Vienna, Vienna, Austria; Department of Zoology, Government \\ College University Lahore, Lahore, Pakistan (M.I.S.); and Department of Pharmaceutical Chemistry, Faculty of Life Sciences, \\ University of Vienna, Vienna, Austria (K.W.)
}

Received March 2, 2017; accepted July 31, 2017

\begin{abstract}
The bile salt export pump (BSEP/ABCB11) transports bile salts from hepatocytes into bile canaliculi. Its malfunction is associated with severe liver disease. One reason for functional impairment of BSEP is systemic administration of drugs, which as a side effect inhibit the transporter. Therefore, drug candidates are routinely screened for potential interaction with this transporter. Hence, understanding the functional biology of BSEP is of key importance. In this study, we engineered the transporter to dissect interdomain communication paths. We introduced mutations in noncanonical and in conserved residues of either of the two nucleotide binding domains and determined the effect on BSEP basal and substrate-stimulated ATPase activity as well as on taurocholate transport. Replacement of the noncanonical methionine residue M584 (Walker B sequence of
\end{abstract}

nucleotide binding site 1) by glutamate imparted hydrolysis competency to this site. Importantly, this mutation was able to sustain $15 \%$ of wild-type transport activity, when the catalytic glutamate of the canonical nucleotide binding site 2 was mutated to glutamine. Kinetic modeling of experimental results for the ensuing M584E/E1244Q mutant suggests that a transfer of hydrolytic capacity from the canonical to the noncanonical nucleotide binding site results in loss of active and adoption of facilitative characteristics. This facilitative transport is ATPgated. To the best of our knowledge, this result is unprecedented in ATP-binding cassette proteins with one noncanonical nucleotide binding site. Our study promotes an understanding of the domain interplay in BSEP as a basis for exploration of drug interactions with this transporter.

\section{Introduction}

ATP-binding cassette (ABC) proteins constitute one of the largest protein families found in all kingdoms of life. In humans, 48 genes encode for $\mathrm{ABC}$ proteins. Most of them are transporters and expressed in tissue-specific manner to serve translocation of diverse cargo across cellular membranes. Malfunction of $22 \mathrm{ABC}$ proteins has been reported to be linked to at least 27 disease etiologies (Chiba et al., 2014). A functional ABC transporter is composed of a minimum of four domains, as follows: two transmembrane domains (TMDs) and two nucleotide binding

This work was supported by grants from the Austrian Science Fund within the scope of SFB35 (to G.S., M.T., T.S., and P.C). M.I.S. is indebted to the Higher Education Commission Pakistan for providing him with a $\mathrm{PhD}$ scholarship to conduct this study.

The corresponding author on behalf of all authors declares that no competing interests exist.

https://doi.org/10.1124/mol.117.108688.

S This article has supplemental material available at molpharm.aspetjournals. org. domains (NBDs). The former confer substrate specificity and provide the translocation paths for ligands, whereas the latter are involved in binding and hydrolyzing ATP, thus providing energy for the active transport of cargo molecules. NBDs contain conserved motifs, including the Walker A and B motifs, ABC signature sequence, and A-, D-, H-, and Q-loops, which are unique to the protein family (Walker et al., 1982; Hyde et al., 1990; Higgins, 1992). These conserved motifs form composite nucleotide binding sites (NBSs). These sites involve the Walker A and B motifs, Q- and H-loop of one, and the signature motif and D-loop of the other NBD (Jin et al., 2012).

The bile salt export pump (BSEP/ABCB11) functions at the canicular membrane of hepatocytes and is responsible for the concentrative transport of bile salts from the hepatocyte cytoplasm into the bile canaliculus. Malfunction of this transporter leads to different disease entities, as follows: 1) progressive familial intrahepatic cholestasis type $2 ; 2$ ) benign recurrent intrahepatic cholestasis; and 3) cholestasis of

ABBREVIATIONS: ABC, ATP-binding cassette; AMP-PNP, 5'-adenylyl-imidodiphosphate; BSEP, bile salt export pump; DMEM, Dulbecco's modified Eagle's medium; HEK, human embryonic kidney; NBD, nucleotide binding domain; NBS, nucleotide binding site; PIC, protease inhibitor cocktail; TMD, transmembrane binding domain. 
pregnancy (Stieger et al., 2007; Kubitz et al., 2012). Furthermore, several systemically administered drugs, among them antidiabetics, antibiotics, endothelin antagonists, and tyrosine kinase inhibitors, compromise BSEP function and thus have the undesired side effect of causing intrahepatic cholestasis (Morgan et al., 2010; Dawson et al., 2012; Pedersen et al., 2013; Ritschel et al., 2014; Montanari et al., 2016).

$\mathrm{BSEP}$ is one of four full-length transporters of the ABCB subfamily. Available crystal structures of type I exporters indicate twofold (C2) rotational symmetry (Dawson and Locher, 2006; Aller et al., 2009; Shintre et al., 2013). In homodimeric half transporters such as ABCB10, binding of ATP at the NBD interface occurs in two rotationally symmetric and otherwise identical NBSs. Similarly, protein architecture necessitates two rotationally symmetric substratebinding modes at the TMD interface. In full transporters (commonly appreciated to have arisen from half transporters by gene duplication) (Chen et al., 1986), the two halves have diverged in the course of evolution, but nevertheless have retained similarity. Our group previously demonstrated that the paradigmatic ABCB transporter P-gp (ABCB1) can bind substrates such as rhodamine 123, verapamil, vinblastine, and propafenones in dual pseudosymmetric mode (Parveen et al., 2011; Dönmez Cakil et al., 2014; Spork et al., 2017). As P-gp has two canonical NBSs, the possibility exists that substrate-binding modes and NBSs function in dedicated manner. In contrast to P-gp, BSEP has only one hydrolysiscompetent NBS (NBS2), which most likely drives taurocholate transport from a single binding site.

We followed the strategy to generate mutants, in which, based on conservation within the ABCB subfamily, noncanonical residues were replaced by canonical ones. We subsequently explored the effect of these mutations on taurocholate transport and ATPase activity. Our data show that selected residues in the noncanonical NBS1 of BSEP are crucial for function, as both transport and ATPase activity decreased, when these residues were replaced. We also mutated conserved lysines of the Walker A motifs and conserved aspartates of the D-loops in both NBS1 and NBS2. Furthermore, we exchanged the catalytic glutamate between NBS2 and NBS1. Most interestingly, our experiments showed that the ensuing mutant M584E/E1244Q retained the ability to transport taurocholate. However, active transport characteristics were lost in this mutant, and, with the help of a kinetic model, we were able to demonstrate that it functions as an ATP-gated facilitator. These experiments allowed us to shed light on the transport mechanism and develop a model for interdomain communication.

\section{Materials and Methods}

Materials. $\left[{ }^{3} \mathrm{H}\right]$ Taurocholate was purchased from American Radiolabeled Chemicals (St. Louis, MO), and Filter Count was purchased from PerkinElmer (Waltham, MA). Sodium taurocholate, $\mathrm{Mg}\left(\mathrm{NO}_{3}\right)_{2}$, DNase I, ATP, cell culture medium, and supplements were bought from Sigma-Aldrich (St. Louis, MO). The protease inhibitor cocktail (PIC) was from Roche (Mannheim, Germany). Chemicals used for buffer preparation, including sucrose, $\mathrm{KCl}, \mathrm{KNO}_{3}$, HEPES, and Tris, were purchased from Roth (Karlsruhe, Germany).

Generation of BSEP Mutants. The following mutations were introduced into human BSEP in the entry vector pENTR4 (Invitrogen, Carlsbad, CA) by using the listed forward and reverse primers (Table 1) (Microsynth, Wolfurt, Austria). The BSEP gene was subsequently transferred from pENTR4 to the proprietary pCEP4d destination vector by using the gateway cloning technology, as described (Hartley et al., 2000). Authenticity of all mutants was confirmed by sequencing of the entire insert in the destination vector. Also, the destination vector was sequenced in its entirety prior to transfer of the insert.

Expression of BSEP in Human Embryonic Kidney 293 Cells. Human embryonic kidney (HEK) 293 cells were transiently transfected with TurboFect (Fisher Scientific, Vienna, Austria), according to the manufacturer's recommendations. Briefly, HEK293 cells were seeded into T25 flasks in Dulbecco's modified Eagle's medium (DMEM) containing $10 \%$ fetal bovine serum and incubated at $37^{\circ} \mathrm{C}$ in an atmosphere containing $5 \% \mathrm{CO}_{2}$. After 24 hours, a mixture of $5 \mu \mathrm{g}$ BSEP containing pCEP4d plasmid and $10 \mu \mathrm{l}$ TurboFect in $500 \mu \mathrm{l}$ DMEM was added to each flask for transfection. Cells were subsequently incubated for another 48 hours, before they were transferred to a T75 flask in selection medium (DMEM, 10\% fetal bovine serum, and $85 \mu \mathrm{g} / \mathrm{ml}$ hygromycin B). After 3-5 days, cells were transferred to T175 flask and used for experiments after another 3-5 days.

Membrane Vesicle Preparation. Plasma membrane vesicles were isolated according to Hirano et al. (2005) and Müller et al. (1994). Eight to 10 days after transfection, cells were harvested, resuspended in $1 \mathrm{ml}$ ice-cold phosphate-buffered saline containing PIC, and centrifuged at $500 \mathrm{~g}$ for 6 minutes at $4^{\circ} \mathrm{C}$. The cell pellet was washed with ice-cold phosphate-buffered saline containing PIC, and again centrifuged at $500 \mathrm{~g}$ for 10 minutes at $4^{\circ} \mathrm{C}$. The pellet was diluted 40-fold with hypotonic buffer ( $1 \mathrm{mM}$ Tris/HCl, $0.1 \mathrm{mM}$ EDTA, pH 7.4) containing PIC and $100 \mathrm{U}$ DNase I (dissolved in $1 \mathrm{ml} 20 \mathrm{mM}$ Tris, $50 \mathrm{mM} \mathrm{NaCl})$ and stirred gently $(\sim 150 \mathrm{~g})$ for 1 hour at $4^{\circ} \mathrm{C}$. Samples were centrifuged at $141,000 \mathrm{~g}\left(30\right.$ minutes, $4^{\circ} \mathrm{C}$ ), and the pellet was resuspended in $4 \mathrm{ml}$ ice-cold isotonic TS buffer $(10 \mathrm{mM}$ Tris/HCl, $250 \mathrm{mM}$ sucrose, $\mathrm{pH}$ 7.4) without PIC. The resuspended pellet was homogenized by 30 strokes with a Dounce B homogenizer (glass/glass, tight pestle). The homogenate was layered over a $38 \%$ (w/vol) sucrose solution in $5 \mathrm{mM}$ Tris/HEPES ( $\mathrm{pH} 7.4$ ), and tubes were centrifuged in an SW28 rotor (L60 ultracentrifuge) at $141,000 \mathrm{~g}$ for 2 hours at $4^{\circ} \mathrm{C}$. The plasma membrane fraction was collected at the gradient interface, diluted to $9 \mathrm{ml}$ with TS buffer, and centrifuged in a fixed angled TLA 100.3 rotor at $100,000 \mathrm{~g}$ for 30 minutes at $4^{\circ} \mathrm{C}$. The pellet was resuspended in $400 \mu \mathrm{l}$ resuspension buffer (50 mM sucrose, $100 \mathrm{mM}$ $\mathrm{KNO}_{3}, 10 \mathrm{mM}$ Tris/HEPES, pH 7.4), and vesicles were formed by passing the solution through a 26-gauge needle 30 times. The membrane vesicles were frozen in liquid nitrogen and stored at $-80^{\circ} \mathrm{C}$ until use. The protein concentration was determined using the Bradford assay with bovine serum albumin as the standard.

Western Blot Analysis. Membrane proteins ( $2.5 \mu \mathrm{g} / \mathrm{lane})$ were separated in a $7.5 \%$ sodium dodecyl sulfate polyacrylamide gel and transferred onto a $0.45-\mu \mathrm{m}$ nitrocellulose membrane (GE Healthcare Life Sciences, Buckinghamshire, UK). The nitrocellulose membrane was incubated overnight at $4^{\circ} \mathrm{C}$ with a mouse monoclonal anti-BSEP antibody [1:1000 dilution, BSEP (F-6); Santa Cruz Biotechnology, Dallas, TX] and a mouse monoclonal anti- $\mathrm{Na}^{+} / \mathrm{K}^{+}$ATPase antibody (1:1000 dilution, M7-PB-E9; Santa Cruz Biotechnology). Then the nitrocellulose membrane was further incubated for 1 hour at room temperature with an anti-mouse secondary antibody, conjugated to horseradish peroxidase (1: 500; GE Healthcare Life Sciences). The protein-antibody complex was detected by using SuperSignal West Pico Chemiluminescence Substrate (Thermo Fischer Scientific, Vienna, Austria), according to the manufacturer's recommendations.

Vesicle Transport Studies. ATP-dependent uptake of taurocholate into isolated plasma membrane vesicles was measured using a rapid filtration technique, as described previously (Gerloff et al., 1998). Vesicles were diluted with resuspension buffer $\left(50 \mathrm{mM}\right.$ sucrose, $100 \mathrm{mM} \mathrm{KNO}_{3}$, $10 \mathrm{mM}$ Tris/HEPES, $\mathrm{pH}$ 7.4) to obtain a concentration of $2.5 \mathrm{mg} / \mathrm{ml}$. A total of $10 \mu \mathrm{l}$ vesicles was prewarmed in a $37^{\circ} \mathrm{C}$ water bath for 30 seconds. Thereafter, taurocholate uptake was initiated by the addition of $40 \mu \mathrm{l}$ uptake buffer [50 mM sucrose, $100 \mathrm{mM} \mathrm{KNO}, 12.5 \mathrm{mM} \mathrm{Mg}\left(\mathrm{NO}_{3}\right)_{2}$, $10 \mathrm{mM}$ Tris/HEPES, $\mathrm{pH}$ 7.4] containing $3 \mu \mathrm{Ci} / \mathrm{ml}\left[{ }^{3} \mathrm{H}\right]$ taurocholate and unlabeled taurocholate to yield a final taurocholate concentration of 
TABLE 1

List of forward and reverse primers used for generation of mutants

\begin{tabular}{|c|c|c|}
\hline Mutations & & Primers \\
\hline \multirow[t]{2}{*}{ M584E } & Forward & $5^{\prime}$-agattctgcttttggacgaggccacctcagctctgg- $3^{\prime}$ \\
\hline & Reverse & $5^{\prime}$-ccagagctgaggtggectcgtccaaaagcagaatct-3' \\
\hline \multirow[t]{2}{*}{ E502S } & Forward & $5^{\prime}$-agatcagattgggatagtgtcgcaagagccagttctgttc- $3^{\prime}$ \\
\hline & Reverse & $5^{\prime}$-gaacagaactggctcttgcgacactatcccaatctgatct- $3^{\prime}$ \\
\hline \multirow[t]{2}{*}{ R1221G and E1223Q } & Forward & $5^{\prime}$-aggggtctcaactctctggagggcagaaacaacgc-3' \\
\hline & Reverse & $5^{\prime}$-gcgttgtttctgectccagagagttgagaccct- $3^{\prime}$ \\
\hline \multirow[t]{2}{*}{$\mathrm{K} 461 \mathrm{M}$} & Forward & $5^{\prime}$-ggacccagtggagctggaatgagtacagcactgcaac- $3^{\prime}$ \\
\hline & Reverse & $5^{\prime}$-gttgcagtgetgtactcattccagctccactgggtcc-3' \\
\hline \multirow[t]{2}{*}{$\mathrm{K} 1119 \mathrm{M}$} & Forward & $5^{\prime}$-ttgttgggagcagtggatgtggcatgagcactagcattc- $3^{\prime}$ \\
\hline & Reverse & $5^{\prime}$-gaatgctagtgctcatgccacatccactgctcccaacaa-3' \\
\hline \multirow[t]{2}{*}{ D590A } & Forward & $5^{\prime}$-ccacctcagctctggccaatgagagtgaagc- $3^{\prime}$ \\
\hline & Reverse & $5^{\prime}$-gcttcactctcattggccagagctgaggtgg- $3^{\prime}$ \\
\hline \multirow[t]{2}{*}{ D1250A } & Forward & $5^{\prime}$-agccacttctgcettagccacagaaagtgaaaagac-3' \\
\hline & Reverse & $5^{\prime}$-gtcttttcactttctgtggctaaggcagaagtggct-3' \\
\hline \multirow[t]{2}{*}{ E1244Q } & Forward & $5^{\prime}$-cgagatcctaaaatcttgctactagatcaggccacttctgcctt- $3^{\prime}$ \\
\hline & Reverse & $5^{\prime}$-aaggcagaagtggectgatctagtagcaagattttaggatctcg-3' \\
\hline
\end{tabular}

$2.5 \mu \mathrm{M}$. It has to be born in mind that substrates of BSEP are detergents. Therefore, taurocholate concentration was carefully selected based on the following rationales: 1) Not exceeding physiologic concentrations; the level of conjugated bile acids in human plasma has been determined by liquid chromatography mass spectrometry to be in the low micromolar range (Bathena et al., 2013). At these concentrations, we thus did not expect damage to occur in our assay system. 2) The concentration of bile acids in wild-type vesicles was found to be about fourfold higher than in the incubation medium and thus would not exceed $10 \mu \mathrm{M}$. 3) In the original publication by Gerloff et al. (1998), which we followed in our experiments, concentrations of taurocholate of up to $15 \mu \mathrm{M}$ were used without evidence for vesicle damage. 4) We do not see a time-dependent deflection of uptake curves over a time period of 80 minutes. This indicates that after reaching a steady state of uptake, an influence of time on vesicle stability is not observed for at least 1 hour at $37^{\circ} \mathrm{C}$. This indicates that vesicle stability is not compromised at the taurocholate concentrations used in our assay system.

The reaction was monitored over time intervals that assured linearity of the reaction at a taurocholate concentration of $2.5 \mu \mathrm{M}$. Transport was stopped by adding $3 \mathrm{ml}$ ice-cold stop solution $(50 \mathrm{mM}$ sucrose, $100 \mathrm{mM} \mathrm{KCl}, 10 \mathrm{mM}$ Tris/HCl, $\mathrm{pH}$ 7.4) into the incubation tube and immediately filtering the mixture through a $0.45-\mu \mathrm{m}$ nitrocellulose acetate filter (Sartorius, Göttingen, Germany), which had been pretreated by passing $3 \mathrm{ml}$ of a $1 \mathrm{mM}$ taurocholate solution through the filter. The tube was rinsed with an additional $3 \mathrm{ml}$ ice-cold stop solution, which was then also filtered. Filters were washed twice with $3 \mathrm{ml}$ ice-cold stop solution and immersed and dissolved in liquid scintillation fluid (Filter Count; PerkinElmer). Vesicle-associated radioactivity was measured in a Packard CA2000 liquid scintillation counter (Packard International, Conroe, TX). To control for nonspecifically bound radioactivity, transport studies were performed in the presence and absence of $5 \mathrm{mM}$ ATP. To monitor taurocholate transport in the presence of ADP and 5'-adenylyl-imidodiphosphate (AMP-PNP), ATP was replaced with these nucleotides in the uptake buffer at identical concentration.

Time Dependence of Taurocholate Transport. For timedependent transport of taurocholate, membrane vesicles $(25 \mu \mathrm{g})$ were incubated in uptake buffer at $37^{\circ} \mathrm{C}$ for $5,10,20,40$, and 80 minutes. Samples were processed, as described above. Curves were fitted to the data points according to the following equation:

$$
y=a \cdot\left(1-e^{-k t}\right)
$$

where $a$ is the difference in accumulation between the zero and infinite time point, $e$ is the Euler number, $k$ is the first order rate constant, and $t$ is the time in minutes.

\section{TABLE 2}

List of residues forming the NBD-NBD contact interface


in this study, are shown in italics. Deviant residues are shown in the fourth and eighth columns.

\begin{tabular}{|c|c|c|c|c|c|c|c|}
\hline Residue in BSEP & Motif & Amino Acids & Alternative Amino Acid (Transporter) & Residue in BSEP & Motif & Amino Acids & $\begin{array}{l}\text { Alternative } \\
\text { Amino Acid } \\
\text { (Transporter) }\end{array}$ \\
\hline \multicolumn{4}{|c|}{ NBD1 } & \multicolumn{4}{|c|}{ NBD2 } \\
\hline 457 & Walker A & $\mathrm{S}$ & $\mathrm{N}(\mathrm{B} 2, \mathrm{~B} 3, \mathrm{~B} 5)$ & 1115 & Walker A & $\mathrm{S}$ & $\mathrm{N}(\mathrm{B} 3)$ \\
\hline 458 & Walker A & $\mathbf{G}$ & & 1116 & Walker A & $\mathbf{G}$ & \\
\hline $502^{a}$ & Q-loop & $\mathbf{S}$ & $\mathrm{G}(\mathrm{B} 2, \mathrm{~B} 3), \mathrm{P}(\mathrm{B} 6, \mathrm{~B} 7), \mathrm{E}(\mathrm{B} 11)$ & 1161 & Q-loop & $\mathbf{Q}$ & \\
\hline 503 & Q-loop & $\mathbf{Q}$ & & 1162 & Q-loop & $\mathrm{E}$ & $\mathrm{D}(\mathrm{B} 6, \mathrm{~B} 7)$ \\
\hline 504 & Q-loop & $\mathrm{E}$ & $\mathrm{D}(\mathrm{B} 6, \mathrm{~B} 7)$ & 1220 & Signature & $\mathbf{S}$ & \\
\hline 560 & Signature & $\mathbf{S}$ & $\mathrm{A}(\mathrm{B} 3)$ & 1221 & Signature & $\mathbf{G}$ & $\mathrm{R}(\mathrm{B} 11)$ \\
\hline 563 & Signature & $\mathbf{Q}$ & $\mathrm{E}(\mathrm{B} 6, \mathrm{~B} 7)$ & 1222 & Signature & $\mathbf{G}$ & \\
\hline 584 & Walker B & $\mathbf{E}$ & $\mathrm{D}(\mathrm{B} 2), \mathrm{M}(\mathrm{B} 11)$ & 1223 & Signature & $\mathbf{Q}$ & $\mathrm{E}(\mathrm{B} 6, \mathrm{~B} 7, \mathrm{~B} 11)$ \\
\hline 587 & D-loop & $\overline{\mathrm{S}}$ & & 1226 & Signature & $\mathrm{R}$ & \\
\hline 588 & D-loop & $\mathrm{A}$ & $\mathrm{S}(\mathrm{B} 7)$ & 1244 & Walker B & $\mathrm{E}$ & \\
\hline 589 & D-loop & $\mathrm{L}$ & & 1247 & D-loop & $\mathrm{S}$ & \\
\hline 590 & D-loop & D & & 1248 & D-loop & $\mathrm{A}$ & $\mathrm{S}(\mathrm{B} 7)$ \\
\hline 615 & H-loop & $\mathbf{H}$ & $\mathrm{Q}(\mathrm{B} 2)$ & 1250 & D-loop & D & \\
\hline 616 & H-loop & $\mathrm{R}$ & $\mathrm{H}$ (B2) & 1275 & H-loop & $\mathbf{H}$ & \\
\hline \multirow[t]{2}{*}{617} & H-loop & $\mathrm{L}$ & & 1276 & H-loop & $\mathrm{R}$ & \\
\hline & & & & 1277 & H-loop & $\mathrm{L}$ & \\
\hline
\end{tabular}

\footnotetext{
${ }^{a}$ Depending on the rotamer, this residue is predicted to interact with either ICL4 or the $\mathrm{Mg}^{2+}$ ion.
} 
Osmolarity Plot. The osmolarity plot was prepared according to Wlcek et al. (2014). Briefly, the membrane vesicles $(25 \mu \mathrm{g})$ were incubated in uptake buffer [50 mM sucrose, $100 \mathrm{mM} \mathrm{KNO}_{3}, 12.5 \mathrm{mM}$ $\mathrm{Mg}\left(\mathrm{NO}_{3}\right)_{2}, 10 \mathrm{mM}$ Tris/HEPES, $\left.\mathrm{pH} 7.4\right]$ at $37^{\circ} \mathrm{C}$ for 10 minutes. Outside osmolarity was increased by adding sucrose [50 $\mathrm{mM}$ (isotonic) to $800 \mathrm{mM}$ ]. Taurocholate transport (picomoles per milligram protein per minute) was plotted against the inverse of the osmolarity. Nonspecific binding of taurocholate to vesicles was calculated by extrapolating the curve to the intercept with the $y$-axis.

ATPase Assay. The ATPase activity was determined by measuring the release of inorganic phosphate using a calorimetric method described by Chifflet et al. (1988) and Schmid et al. (1999). Briefly, $25 \mu \mathrm{g}$ membrane vesicles was added to the ATPase assay buffer ( $300 \mathrm{mM}$ sucrose, $4.8 \mathrm{mM}$ $\mathrm{MgCl}_{2}, 0.2 \mathrm{mM}$ EGTA, $10 \mathrm{mM} \mathrm{NaN}, 0.17 \mathrm{mM}$ ouabain, and $20 \mathrm{mM}$ Tris/HCl, $\mathrm{pH}$ 7.4). Ouabain, EGTA, and $\mathrm{NaN}_{3}$ were added in the assay mixture to block $\mathrm{Na}^{+} / \mathrm{K}^{+}, \mathrm{Ca}^{2+}$, and mitochondrial ATPases, respectively. Orthovanadate $(0.56 \mathrm{mM})$ was used to block $\mathrm{ABC}$ transporterassociated ATPase activity. BSEP-associated ATPase activity was calculated as the difference of total and vanadate-insensitive ATPase activity. The latter had to be corrected for vanadate-sensitive ATPase activity in mock-transfected cells. For ATPase stimulation, taurocholate was used at a concentration of $50 \mu \mathrm{M}$. The assay mixture was prewarmed to $37^{\circ} \mathrm{C}$ for 5 minutes, and the reaction was started by addition of ATP ( $4 \mathrm{mM}$ ). After 10, 20, 30, and 40 minutes, a $50 \mu \mathrm{l}$ aliquot of the incubation mixture was added to $50 \mu \mathrm{l} 12 \%$ SDS in assay buffer (in 96-well microtiter plate) to stop the reaction. For color development, $100 \mu \mathrm{l}$ freshly prepared $6 \%$ ascorbic acid in $1 \mathrm{M} \mathrm{HCl}$ and $1 \%$ ammoniumheptamolybdate was added, and plates were incubated at room temperature for 5 minutes. Subsequently, $150 \mu \mathrm{l} 2 \%(\mathrm{w} / \mathrm{v})$ trisodium citrate and $2 \%(\mathrm{w} / \mathrm{v})$ sodium (meta)arsenite in $2 \%(\mathrm{v} / \mathrm{v})$ acetic acid were added, and the mixture was incubated at room temperature for 20 minutes to stabilize and enhance the color. The optical density was measured at $630 \mathrm{~nm} . \mathrm{Na}_{2} \mathrm{HPO}_{4}$ (stock $2 \mathrm{mM}$ ) was used to prepare the standard curve.

Homology Modeling of BSEP. The sequences of ABCB1, BSEP, and Sav1866 were aligned and manually adjusted, according to Stockner et al., (2009). Models of BSEP were created with MODELER version 9.15 (Sali and Blundell, 1993) using the crystal structure of Sav1866 from Staphylococcus aureus as the template (PDB ID: 2ONJ) (Dawson and Locher, 2007). Mutations of residues in NBS1 were introduced with MODELER. The extracellular loop 1 was modeled to continue $\alpha$-helicity of transmembrane helices 1 and 2, based on secondary structure predictions. The cocrystallized nucleotide AMP-PNP was replaced by ATP; $\mathrm{Na}^{+}$ions in the crystal structure were replaced by $\mathrm{Mg}^{+2}$ ions.

Kinetic Modeling. Taurocholate uptake and ATPase activity of BSEP-containing plasma membrane vesicles were simulated according to a previously published kinetic model of the ABCB1 transport cycle (Al-Shawi et al., 2003) with modifications. The geometry of the vesicles was assumed to be spherical with a diameter of $2 \mu \mathrm{m}(\mathrm{V}=4.18 \mathrm{fl})$. Loss of taurocholate from vesicles to the incubation medium was taken into account in the model. Permeability of taurocholate was estimated on basis of the physicochemical property "polar surface area," as described (Schmid et al., 2015). Time-dependent changes in state occupancies, as well as substrate efflux and phosphate generation, were evaluated by numerical integration of the resulting system of differential equations using the Systems Biology Toolbox (Schmidt and Jirstrand, 2006) and MATLAB 2012a (Mathworks, Natick, MA).

Statistical Analysis. The GraphPad prism software (GraphPad Software, La Jolla, CA) was used for data analysis. Statistically significant differences between basal and taurocholate-stimulated ATPase activity were determined by two-way analysis of variance, followed by Bonferroni post hoc analysis. For comparison of taurocholate transport, one-way analysis of variance, followed by Tukey's post hoc analysis, was used. A $P$ value $<0.05$ was considered to be statistically significant. A comparison of the goodness of fit for a linear and nonlinear fit was performed using the extra sum-of-squares $F$ test. To be able to perform an extra sum-of-squares $F$ test, we alternatively fitted data points not only with the kinetic model, but also with a quadratic function, according to the equation $y=a^{2}+b x+c$.
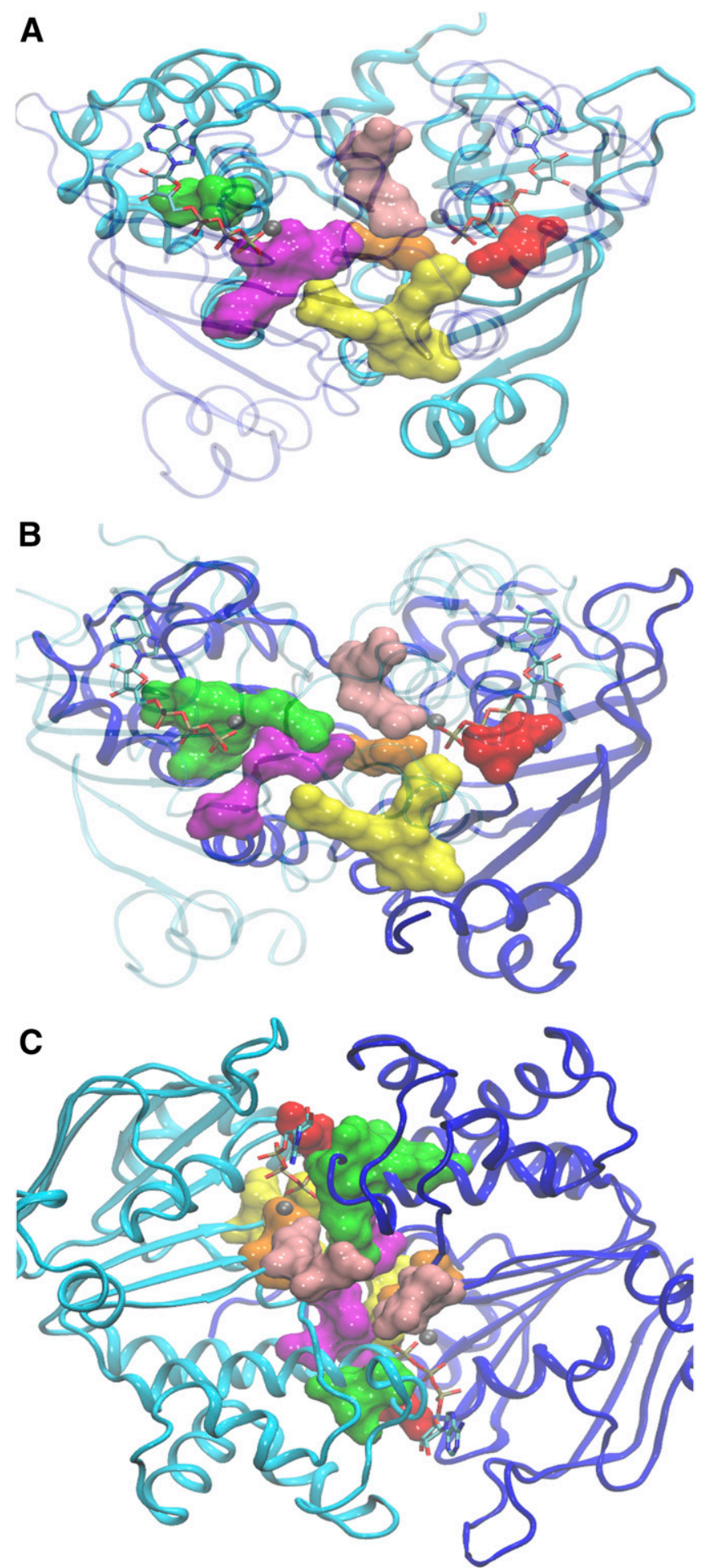

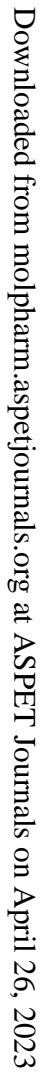

Fig. 1. Homology model of BSEP based on the crystal structure of Sav1866. NBD1 and NBD2 are shown in ribbon representation in cyan and blue, respectively. Residues that are in direct contact at the interface of the NBDs are rendered in surface representation (Walker A motif, red; Q-loop, pink; signature sequence, green; Walker B, orange; D-loop, magenta; H-loop, yellow). The two ATP molecules are shown in licorice rendering. $\mathrm{Mg}^{2+}$ ions are depicted as gray spheres: (A) Side view of the NBD1 interface with a view angle that runs perpendicular to the plane of the interface. (B) Side view of the NBD2 interface [rotated by $180^{\circ}$ relative to (A)]. (C) Top view with a view angle that runs along the NBD interface. 


\section{Results}

Role of Noncanonical Residues in NBS1 for Taurocholate Transport and BSEP ATPase Activity. Two ATP molecules are bound and sandwiched between the two NBDs of ABC proteins in two composite NBSs, NBS1 and NBS2. The two NBDs form direct contacts with each other by contribution of residues, which are listed in Table 2 and shown in a homology model of BSEP in Fig. 1. Contact residues are defined as those residues in one NBD, which can come in contact with residues in the other NBD. Some of these are also in contact with the ATP molecules. Figure 1 shows contact residues in surface rendering for NBD1 (Fig. 1A) and NBD2 (Fig. 1B) in a head-on view onto the respective NBD and in a top view for both NBDs (Fig. 1C). All contact residues lie close, or conform to conserved residues of NBD motifs in the primary sequence. All four full transporters of the ABCB subfamily show complete contact-interface conservation, except for four residues in BSEP, which all lie in the noncanonical NBS1 (Fig. 2).

To assess the role of these four amino acid residues and to establish the functional relevance of asymmetry in the NBSs, we mutated them to conform to those, which are found in other members of the ABCB subfamily (E502S/M584E/R1221G/ $\mathrm{E} 1223 \mathrm{Q}$ ). In addition to the quadruple, we also created a single mutant, in which the methionine of the NBD1 Walker B motif was replaced by glutamate (M584E). Taurocholate transport activity was evaluated in inside-out membrane vesicles prepared from HEK293 cells expressing wild-type or mutant BSEP. Expression was determined by Western blotting and found to be similar in wild-type and mutants. A representative immunoblot is shown in Fig. 3A. The column graph in Fig. 3B

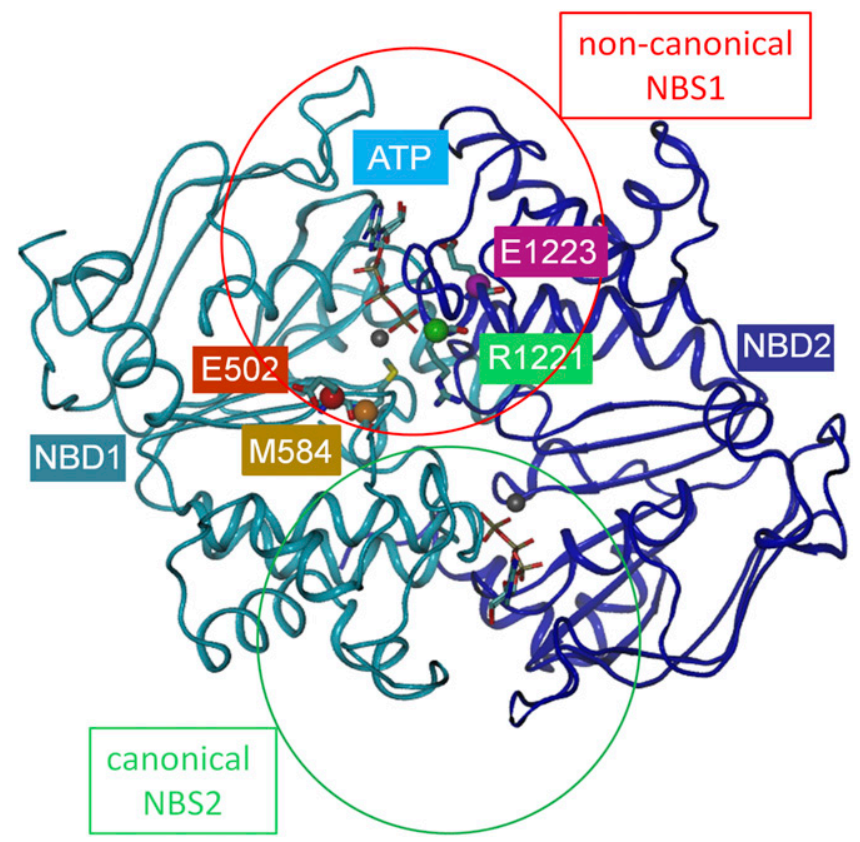

Fig. 2. Homology model of BSEP based on the crystal structure of Sav1866. The N-terminal NBD is shown in cyan, and the C-terminal NBD in blue color. C- $\alpha$ atoms of noncanonical residues of NBS1 are shown as spheres, and side chains are in licorice rendering. Residue E502 is shown in red, M584 in orange, R1221 in green, and E1223 in magenta. NBS1 and NBS2 are indicated by red and green circles, respectively. Magnesium ions are depicted as gray spheres and located in close proximity of the $\beta$ and $\gamma$ phosphate of two ATP molecules. gives results from repeat experiments, and Fig. $3 \mathrm{C}$ shows the corresponding transport data. The Walker B single mutant (M584E), in which hydrolysis competency was imparted to NBS1, showed approximately $90 \%$ of wild-type transport activity. In the quadruple mutant, in which the four deviant amino acid residues in NBS1 were replaced by canonical residues (as found in other ABCB subfamily members), transport was reduced to about $35 \%$ (Fig. 3C).
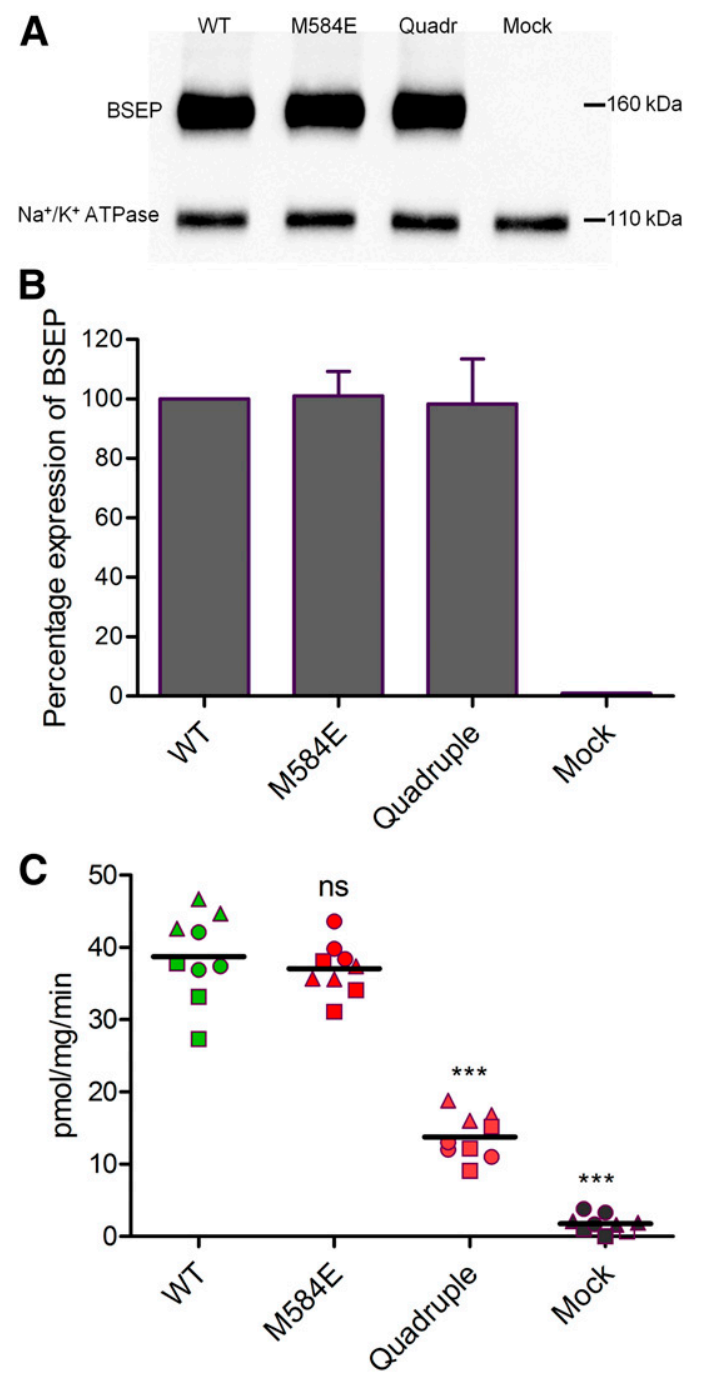

Fig. 3. (A) Expression of BSEP was determined by using the monoclonal BSEP-F6 antibody. $\mathrm{Na}^{+} / \mathrm{K}^{+}$ATPase was used as a loading control. Vesicles from mock-transfected cells served as the negative control. (A) Western blot of one representative experiment is shown. (B) Column graph for relative band intensities (mean \pm S.D.) of three independent experiments. Expression of wild-type was taken as $100 \%$. (C) ATP-dependent transport of $\left[{ }^{3} \mathrm{H}\right]$ taurocholate into inside-out membrane vesicles containing wildtype (WT); NBS1 mutants are shown along with negative controls (mock). Taurocholate transport was measured, as described in Materials and Methods. BSEP-mediated transport was calculated by subtracting transport activity in the absence of ATP from that measured in the presence of ATP. Data points conform to triplicates from three independent experiments (three independently prepared vesicle batches), which are shown as filled circles, triangles, and squares. Horizontal bars indicate mean values. To calculate statistical differences between the groups, averages of individual experiment $(n=3)$ were subjected to one-way analysis of variance, followed by Tukey's post hoc test to compare more than one group with wild-type. Statistical significance levels are indicated by asterisks $(* * * P<$ 0.001). ns, No statistical significance. 
As ATP binding and hydrolysis is a prerequisite for transport function, we also measured basal and taurocholate-stimulated ATPase activity of the mutants. Two negative controls were used in these experiments: 1 ) vesicles from mock-transfected cells and 2) cells transfected with the E1244Q mutant. This mutant lacks a catalytic glutamate and thus is unable to either hydrolyze ATP or transport taurocholate (see below). Vesicle preparations from these negative controls showed vanadatesensitive ATPase activity of about $3 \mathrm{nmol} \mathrm{P}_{\mathrm{i}} / \mathrm{mg}$ per minute. This ATPase activity is not BSEP associated as it is not stimulated by taurocholate (Fig. 4).

Basal BSEP ATPase activity of the M584E mutant was comparable to that of wild-type protein. ATPase and transport activity in the quadruple mutant was decreased to about $35 \%$. ATPase stimulation by substrate was retained in both the single and the quadruple mutant. This indicates that directional communication between the taurocholate binding site in the TMDs and the hydrolysis-competent NBS2 is retained despite the introduction of these mutations in NBS1.
Exploring the Role of Canonical Residues in NBS1 and NBS2. Despite the presence of individual noncanonical residues, most of the residues in NBS1 are conserved within the ABCB subfamily. These include the lysine residue in the Walker A motif and the aspartate residue in the D-loop. Hence, we explored the effect of mutation of these residues on taurocholate transport and ATPase activity in NBS1. As a control, we also introduced these mutations in NBS2.

Role of Walker A Lysine Residues. Walker A lysines were shown to form contacts with the $\beta$ and $\gamma$ phosphate of ATP (Hung et al., 1998). We replaced these lysines by methionines (K461M and $\mathrm{K} 1119 \mathrm{M})$, as previously described for ABCB1 (Lapinski et al., 2001; Bársony et al., 2016). Surface expression of these mutants was found to be comparable to that of wild-type protein (Fig. 5, A and B). Taurocholate transport was found to be abolished in both mutants (Fig. 5C), whereas a basal BSEP-associated ATPase activity was still found to be associated with the mutants. The K1119M mutant lost its ability to be stimulated by taurocholate (Fig. 4).

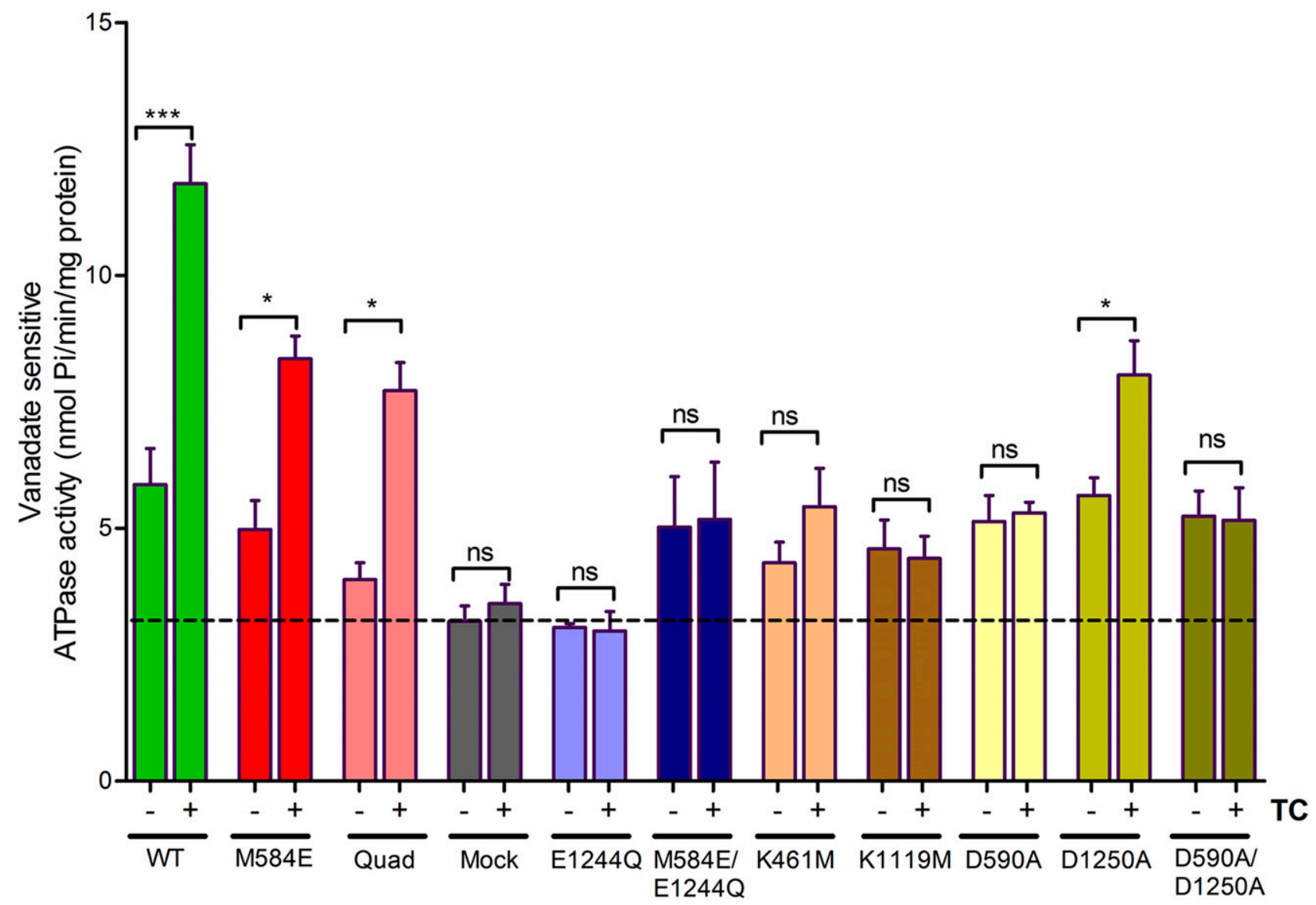

Fig. 4. ATPase activity was measured in inside-out plasma membrane vesicles prepared from HEK293 cells transfected with wild-type or mutant BSEP. Generally, orthovanadate is used to determine BSEP-associated ATPase activity. Mock-transfected cells and cells transfected with the ATPase-deficient E1244Q mutant were used as negative controls (gray and light blue bars, respectively). The black dotted line shows the proportion of ATPase activity, which is not BSEP associated. It is due to endogenous vanadate-inhibited ATPases, which are present in the plasma membrane of HEK293 cells. For ATPase stimulation, taurocholate (TC) was used at a final concentration of $50 \mu \mathrm{M}$. This exceeds the concentration required for half-maximal stimulation in wild-type protein by four- to fivefold (Supplemental Fig. 1). Stimulation here was taken as a qualitative parameter for interdomain communication. Fold stimulation thus is not intended to provide quantitative information, as curves for concentration dependencies might be shifted in mutants relative to wild-type. The number of experiments was three for all mutants (quadruplicate determinations from independent incubation mixtures). In individual experiments, these mutants were put together in different combinations, whereby wild-type was always included as a positive control. Thus, $n$ was 3 for all mutants, but 13 for wild-type. Mean values \pm S.D. are shown. Statistically significant differences between basal and taurocholate-stimulated ATPase activity were determined by two-way analysis of variance, followed by Bonferroni post hoc analysis. Statistical significance levels are indicated by asterisks $(* P<0.05 ; * * * P<0.001)$. ns, no statistical significance. 
These results suggest that communication between the taurocholate binding site and NBS2 depends on the presence of this conserved lysine residue in NBS2.

Role of Conserved D-Loop Aspartates. In an additional series of experiments, we mutated D-loop aspartates in NBS1 and NBS2, because of their previously reported role in NBS-NBS communication (Hohl et al., 2014). Surface expression was comparable to that of wild-type (Fig. 6, A and B). Mutation of aspartate (D590) in canonical NBS2 to alanine decreased transport activity to the level observed in mock-transfected cells. In contrast, mutation of the analogous
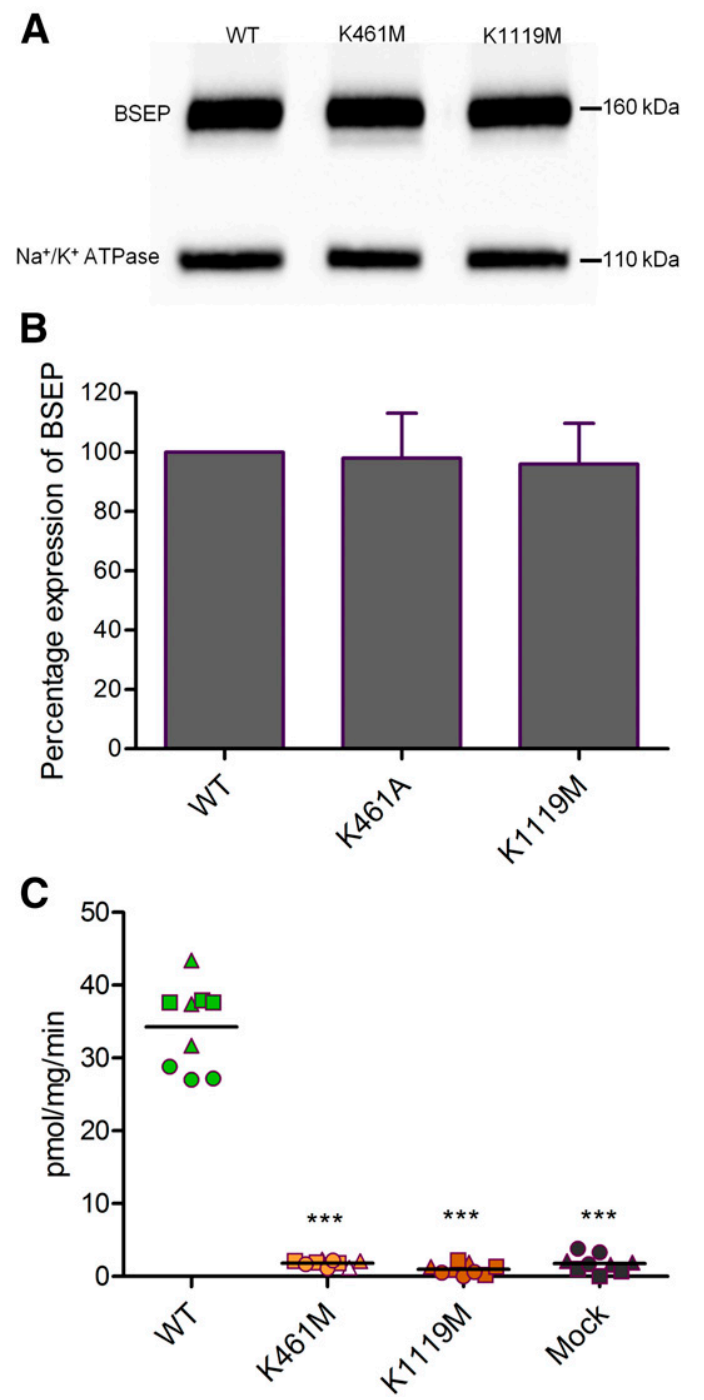

Fig. 5. (A) Expression of BSEP was evaluated by using the monoclonal BSEP (F-6) antibody. $\mathrm{Na}^{+} / \mathrm{K}^{+}$-ATPase was used as a loading control. (A) Representative Western blot for one experiment. (B) Column graph for relative band intensities (mean \pm S.D.) of three independent experiments. Expression of wild-type was taken as $100 \%$. (C) ATP-dependent transport of $\left[{ }^{3} \mathrm{H}\right]$ taurocholate into inside-out membrane vesicles containing wildtype or Walker A mutants. Taurocholate transport was measured, as described in Materials and Methods. BSEP-mediated transport was calculated by subtracting transport values in the absence of ATP from the values measured in the presence of ATP. Data points conform to triplicates from three independent experiments, which are shown as filled circles, triangles, and squares. Horizontal bars indicate mean values. To calculate statistical differences between the groups, averages of individual experiment ( $n=3$ ) were subjected to one-way analysis of variance, followed by Tukey's post hoc test. Statistical significance levels are indicated by asterisks $(* * * P<0.001)$. aspartate residue in NBS1 to alanine (D1250A) resulted in a reduction of transport activity to about $50 \%$ of wild-type. The double-mutant D590A/D1250A was inactive (Fig. 6C). Basal

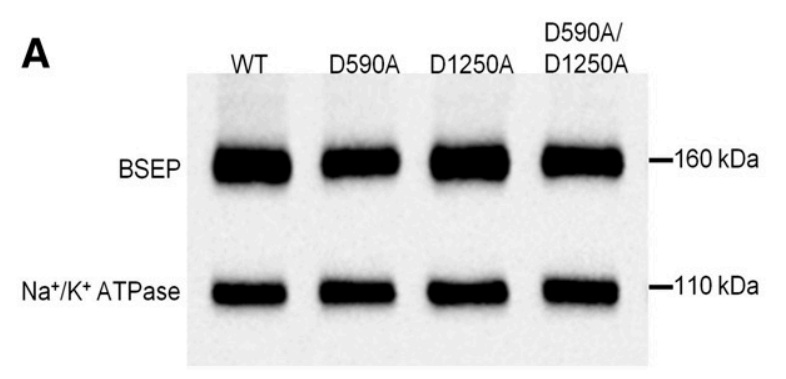

B
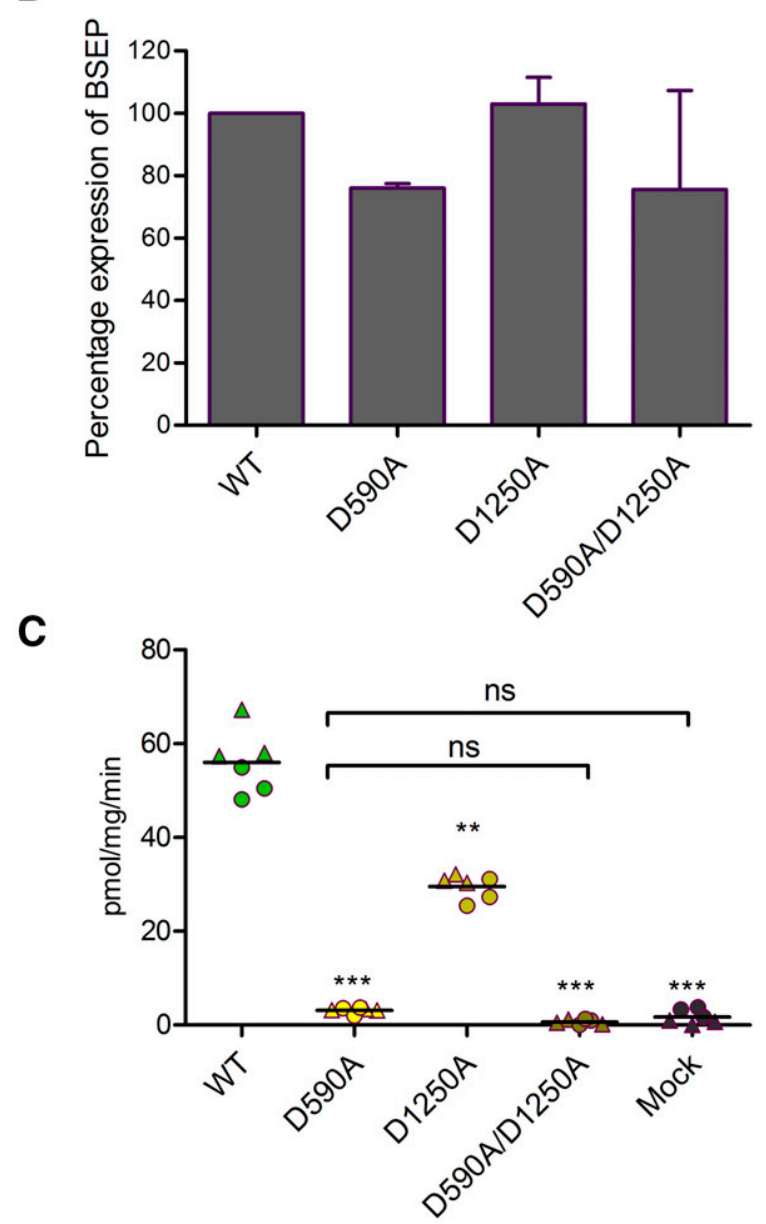

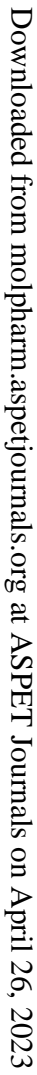

Fig. 6. (A) Expression of BSEP was evaluated by using the monoclonal $\mathrm{BSEP}$ (F-6) antibody. $\mathrm{Na}^{+} / \mathrm{K}^{+}$-ATPase was used as a loading control. In (A), a representative Western blot for one experiment is shown. (B) Column graph comparing relative band intensities (mean \pm S.D.) of two independent experiments. Expression of wild-type was taken as $100 \%$. (C) ATP-dependent transport of $\left[{ }^{3} \mathrm{H}\right]$ taurocholate into inside-out membrane vesicles containing wild-type or D-loop mutants is shown. Taurocholate transport was measured, as described in Materials and Methods. BSEPmediated transport was calculated by subtracting transport in the absence of ATP from that measured in the presence of ATP. Data points conform to triplicates from two independent experiments, which are shown as filled circles and triangles. Horizontal bars indicate mean values. To calculate statistical differences between the groups, averages of individual experiment $(n=2)$ were subjected to one-way analysis of variance, followed by Tukey's post hoc test. Statistical significance levels are indicated by asterisks $(* * P<0.01 ; * * * P<0.001)$. The absence of statistical significance between negative control (mock) and the D590A and the D590A/D1250A mutant is indicated by brackets and ns (not statistically significant). 
ATPase activity in the single and the double mutant was comparable to that of wild-type protein. NBS1 mutant D1250A was stimulated by taurocholate. However, this stimulation was absent in the D590A (NBS2) and in the double-mutant D590A/ D1250A (Fig. 4).

Exchanging the Catalytic Glutamate between NBS2 and NBS1 Retains Transport Activity. The catalytic glutamate of the Walker B motif is involved in ATP hydrolysis.
In BSEP, only the Walker B motif of NBS2 contains a conserved glutamate, although the analogous residue in NBS1 is a methionine. Therefore, NBS2 is capable of ATP hydrolysis, whereas NBS1 is not. We first mutated the catalytic glutamate of NBS2 to glutamine (E1244Q) to render it hydrolysis incompetent. Again, plasma membrane expression of this mutant was comparable to that of wild-type protein (Fig. 7, A and B). This mutant was transport deficient as its
A

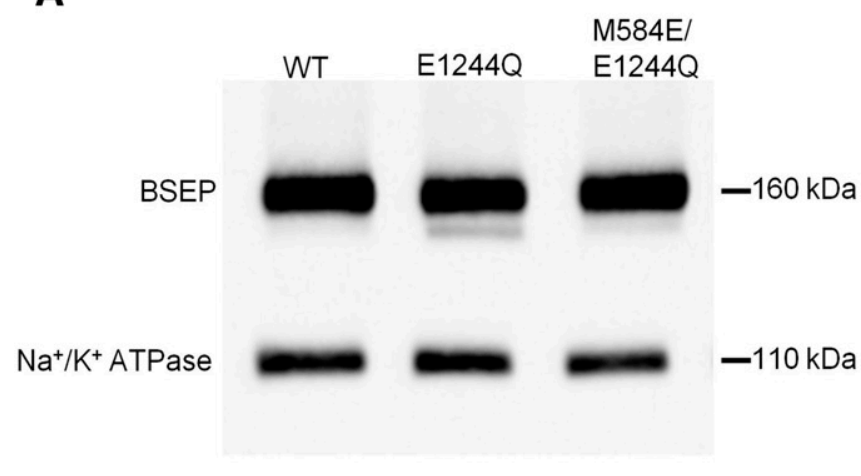

B

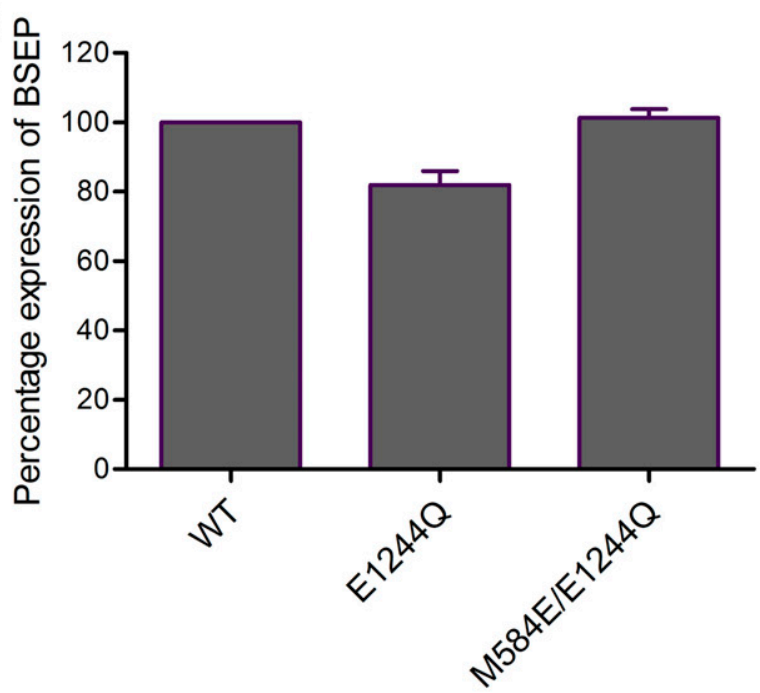

D

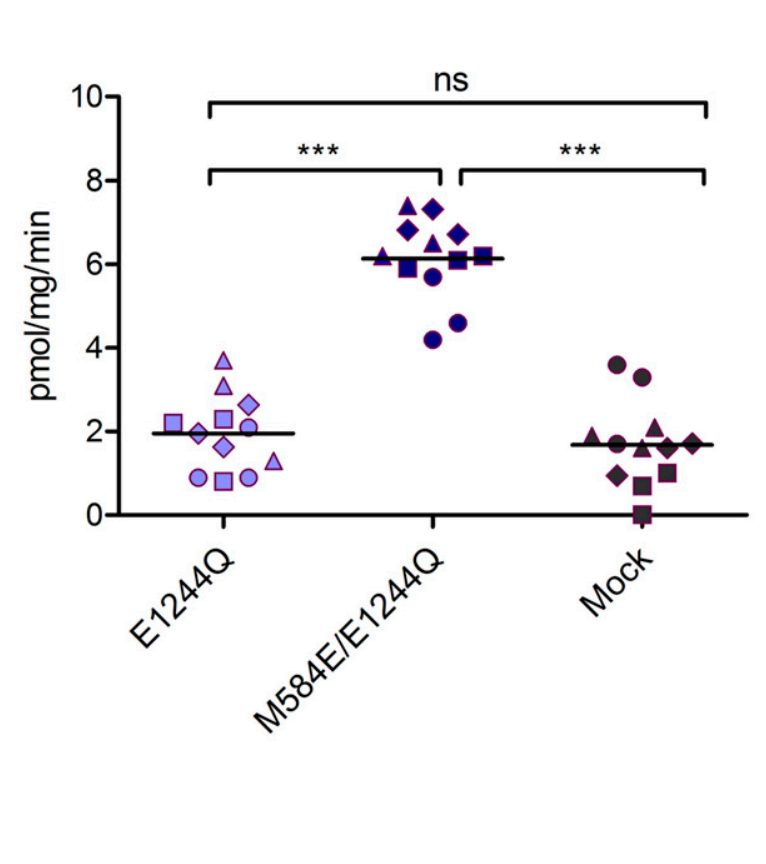

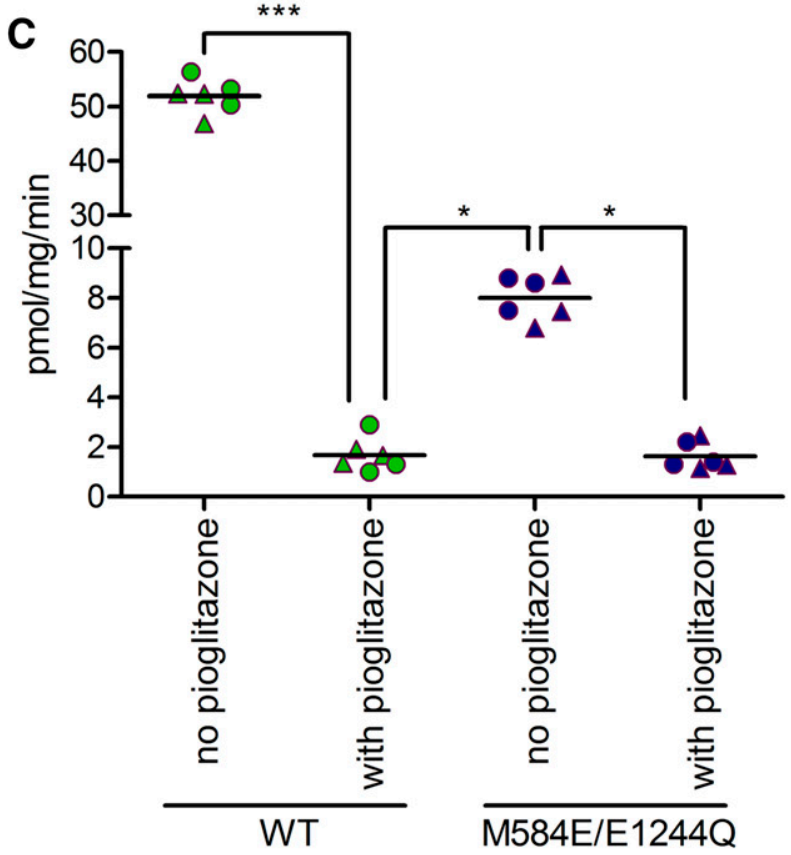

Fig. 7. (A) Expression of BSEP was evaluated by using the monoclonal BSEP (F-6) antibody. $\mathrm{Na}^{+} / \mathrm{K}^{+}-\mathrm{ATP}$ ase was used as a loading control. In (A), a representative Western blot for one experiment is shown. (B) Column graph comparing relative band intensities (mean \pm S.D.) of four independent experiments. Expression of wild-type was taken as 100\%. (C) ATP-dependent taurocholate transport in the absence and presence of $10 \mu \mathrm{M}$ pioglitazone, a potent inhibitor of BSEP. Pioglitazone inhibited transport in both wild-type and the M584E/E1244Q mutant, indicating that taurocholate transport is BSEP mediated. Data points conform to triplicates from two independent experiments, which are shown as filled circles and triangles. Horizontal bars indicate mean values. To calculate statistical differences between the groups, averages of individual experiment $(n=2)$ were subjected to one-way analysis of variance, followed by Tukey's post hoc test. Statistical significance levels are indicated by asterisks $(* P<0.05$; $* * * P<0.001)$. (D) ATPdependent transport of $\left[{ }^{3} \mathrm{H}\right]$ taurocholate into inside-out membrane vesicles containing wild-type, the E1244Q, or the M584E/E1244Q mutant. Taurocholate transport was measured, as described in Materials and Methods. BSEP-mediated transport was calculated by subtracting transport values in the absence of ATP from the values measured in the presence of ATP. Data points conform to triplicates from four independent experiments, which are shown as filled circles, triangles, squares, and diamonds. Horizontal bars indicate mean values. To calculate statistical differences between the groups, averages of individual experiment $(n=4)$ were subjected to one-way analysis of variance, followed by Tukey's post hoc test. Statistical significance levels are indicated by asterisks $(* * * P<0.001)$. 
transport activity was not significantly different from that found in mock-transfected cells. Also, ATPase activity was comparable to that found in mock-transfected cells (Fig. 4). Thus, any BSEP-associated ATPase activity was absent.

We next replaced the noncanonical methionine residue in NBS1 with glutamate in the background of the E1244Q mutant. The catalytic glutamate was thus exchanged between NBS2 and NBS1 (M584E/E1244Q) to reinstate hydrolysis competency in the protein. The mutant again showed a surface expression that was comparable to that of wild-type protein (Fig. 7, A and B). Most interestingly, the double mutant consistently showed approximately $15 \%$ residual transport activity (Fig. 7C), which was demonstrated to be BSEP mediated by using the highaffinity inhibitor pioglitazone [published $\mathrm{IC}_{50}$ values of $0.4 \mu \mathrm{M}$ (Morgan et al., 2010) and $0.3 \mu \mathrm{M}$ (Dawson et al., 2012)]. At a concentration of $10 \mu \mathrm{M}$ pioglitazone, transport was completely abolished in the mutant and in wild-type (Fig. 7C). This residual transport activity by the M584E/E1244Q mutant was significantly higher than that of the catalytically inactive mutant E244Q and mock (Fig. 7D). To further characterize this mutant, we monitored taurocholate transport in the absence of nucleotide and in presence of ATP, ADP, and AMP-PNP. Similar to wildtype, the mutant showed strict ATP dependence of transport activity (Fig. 8).

We further determined basal ATPase activity of the mutant and the ability of taurocholate to stimulate it (Fig. 4). Basal activity was comparable to wild-type, indicating that the M584E/E1244Q mutant was capable of hydrolyzing ATP. As NBS2 in this mutant is hydrolysis incompetent, this activity must be mediated by NBS1. However, stimulation of ATPase activity by taurocholate was not observed. Hence, the taurocholate binding site cannot signal the presence of substrate to NBS1. Because the mutant is capable of taurocholate transport, this lack of substrate stimulation is not considered to be brought about by a loss of the ability to bind taurocholate.

To further explore the molecular mechanism of taurocholate transport by the M584E/E1244Q mutant, we compared steady state levels of uptake in the mutant and wild-type protein.

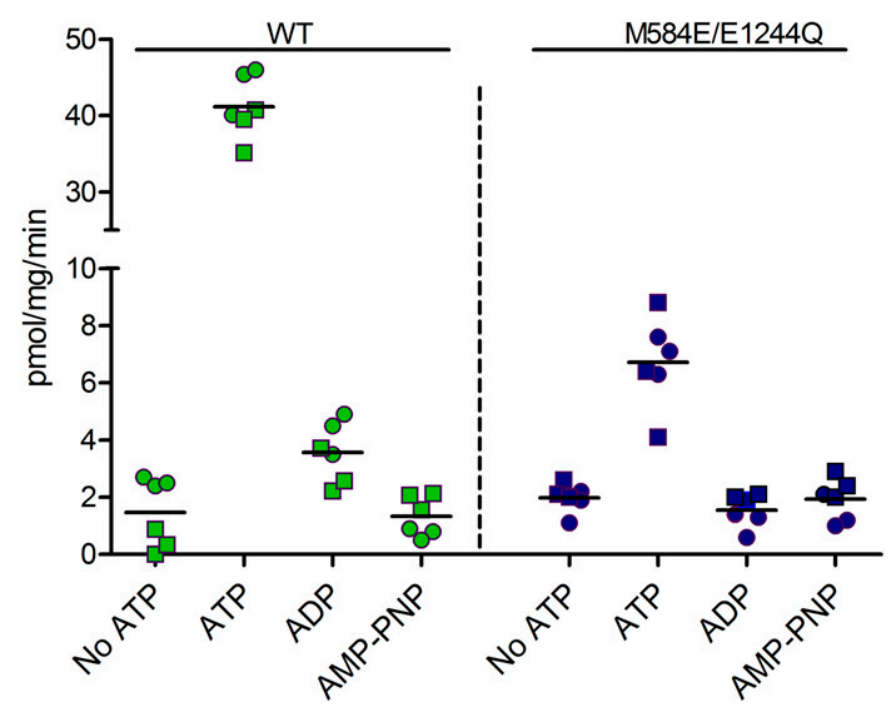

Fig. 8. The transport of taurocholate $(2.5 \mu \mathrm{M})$ was monitored in the presence of $5 \mathrm{mM}$ ATP, ADP, or AMP-PNP. Data points conform to triplicates from two independent experiments from two separately prepared vesicle batches. The horizontal bar indicates the mean value of data points.
Uptake of taurocholate was monitored over 80 minutes at a concentration of $2.5 \mu \mathrm{M}$ (first order) (Fig. 9). Steady state levels of uptake were more than four times lower in the mutant (approximately $170 \mathrm{pmol} / \mathrm{mg}$ ) as compared with wildtype (approximately $700 \mathrm{pmol} / \mathrm{mg}$ ), and transport velocity was reduced (half-life of accumulation 5.1 minutes in wild-type and 14.6 minutes in the mutant). Despite similar basal ATP hydrolysis rates, steady state levels of taurocholate accumulation were thus reduced in the mutant.

Taurocholate is an amphipathic small molecule, which readily distributes into the environment of biomembranes. To discriminate between biomembrane partitioning and accumulation in the vesicle lumen, transport was monitored in the presence of an increasing concentration of sucrose in the uptake buffer. This collapses vesicles (Supplemental Fig. 2). Transport rate is expected to be linearly related to the inverse of the osmolarity, where the intercept with the ordinate conforms to vesicle-associated radioactivity at zero inside volume. Under the assumption that binding of taurocholate to the transporter does not significantly contribute to this value, the intercept of wild-type and mutant should be identical.

Figure 10 shows that the mutant indeed demonstrates linear behavior. In contrast, data points for wild-type transporter seem to deviate from linearity. When fitted linearly, the intercept value is much higher than for the mutant. A similar deviation of data points from linear fits can be observed in an experimental setup that is comparable to ours (Wlcek et al., 2014). To resolve the discrepancy of nonconforming $y$-intercepts for wild-type protein and mutant, we simulated the process of taurocholate accumulation in vesicles by using a kinetic BSEP model (Supplemental Fig. 3A). Briefly, this model is based on the following assumptions: one molecule of ATP is bound to the transporter in NBS1 in the inward-facing conformation. Substrate binding induces binding of a second molecule of ATP in NBS2. This brings the transporter into an outward-facing conformation. Subsequent hydrolysis of ATP in NBS2 leads to substrate release. In two additional steps, phosphate and ADP are released. This resets the transporter

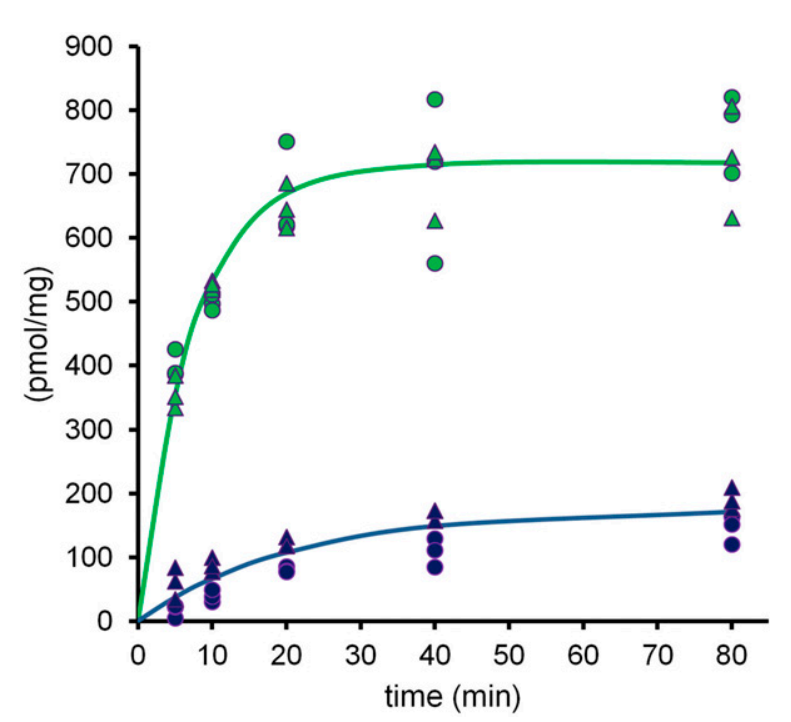

Fig. 9. Time dependence of taurocholate uptake in inside-out membrane vesicles containing wild-type BSEP (green) or the M584E/E1244Q mutant (blue). Data points conform to triplicates from two independent experiments from two separately prepared vesicle batches. 




Fig. 10. ATP-dependent taurocholate transport in wild-type (green) and the M584E/E1244Q mutant (blue) at different osmolarity (Osm). The latter was increased by adding sucrose to the incubation medium. Experimentally determined taurocholate transport rates (filled circles) were plotted as a function of the inverse of the osmolarity. The dashed green line is a linear fit of data points for wild-type BSEP. Solid lines correspond to a nonlinear fit based on the kinetic model shown in Supplemental Fig. 3. If only data points for the five different osmolarities are considered, a significant difference between the two fits (linear versus nonlinear) is not observed. Importantly, an additional constraint has to be taken into consideration. This constraint is defined by the inability of the transporter to accumulate substrate in vesicles with zero inside volume. When considering this constraint, a nonlinear fit (solid green line) performs significantly better than a linear fit (dotted green line, $P=0.0001$, extra sum of squares $F$ test). Data points in the absence of ATP are depicted as dot-dashed lines (wild-type: down triangles; M584E/E1244Q mutant: squares). These data points represent the sum of unspecific membrane association of taurocholate and binding to BSEP and define the intersection point of curves obtained by the kinetic model.

to the inward-facing conformation. The second molecule of ATP can also bind in the absence of substrate and has to undergo hydrolysis to return the transporter to a substrate-binding competent state. This empty cycle satisfies the observation that basal ATPase activity is observed in the absence of taurocholate.

Because of the fact that removal of ATP and substrate from the medium led to progressive loss of previously accumulated taurocholate from the vesicles, we also allowed for a concentration gradient-driven loss across the vesicle membrane. As laid out in Materials and Methods, this loss of taurocholate into the vesicle environment is not due to detergent action of the substrate. Estimates for diffusion rates were obtained experimentally by diluting wild-type vesicles 60-fold at steady state (data not shown). We also devised a model for a facilitator (Supplemental Fig. 3B). Once again, one ATP is bound to the transporter, but this time to NBS2 rather than to NBS1. Binding of a second molecule of ATP generates the outward-facing conformation. In the presence of substrate, the quaternary complex $\mathrm{ATP}_{2}-\mathrm{T}_{\mathrm{e}}-\mathrm{S}$ is thus shared by wild-type protein and mutant. Substrate dissociates at lower rate than in wild-type, in which this process is promoted by ATP hydrolysis in NBS2. The ATP molecule in NBS2 can now leave the transporter without being hydrolyzed, but only when hydrolysis in NBS1 takes place. The following evidence supports this notion: 1) transport is abolished when the protein is rendered hydrolysis incompetent (mutation of E1244 to glutamine) and 2) transport is abolished in the presence of AMP-PNP.

Parameters and constants were numerically determined by solving the kinetic models and found to be close to those values that were initially entered into the equations as estimates (Supplemental Table 1). Models are able to accurately predict all experimental data, not only those from osmolarity plots, but also time dependencies, concentration-dependent stimulation of ATPase activity, and, last but not least, the phenomenon of basal BSEP ATPase activity. Solid lines in Fig. 10 represent modeling data for wild-type (green) and mutant (blue), whereas filled symbols depict the experimentally determined data points. Clearly, curves generated by the kinetic model show excellent agreement with experimentally determined data points. Physiologically, BSEP is capable of concentrating bile acids in bile canaliculi (Cuperus et al., 2014). For wild-type protein, this causes a concentration gradient from the inside of the vesicle to the outside, which promotes loss of vesicle-accumulated taurocholate back into the medium. In a mutant with facilitative transport characteristics, a gradient between the inside and the outside of the vesicle cannot be generated, and thus, outward diffusion is not observed. In this study, a linear behavior is predicted and experimentally found. In contrast, in wild-type the active transporter generates a concentration gradient, which causes outward diffusion that is dependent on vesicle volume. In this work, a downward deflection of the curve is observed with increasing osmolarity (from right to left).

\section{Discussion}

Homodimeric half transporters, as well as a number of fulllength transporters, have two hydrolysis-competent NBSs. In ABCB1 two canonical ATP binding sites find a counterpart in dual substrate-binding modes. This duality is rooted in the rotationally symmetric architecture of the protein (Parveen et al., 2011). However, noncanonical residues are present in the NBS1 of about $40 \%$ of human ABC proteins (Procko et al., 2009). In these transporters, symmetry is lost and complexity of the catalytic cycle is reduced. With respect to mechanistic details of ATP binding to a noncanonical NBS1, the following possibilities exist: 1) ATP binds to NBS1 for prolonged periods of time and holds the NBDs together, whereas NBS2 hydrolyzes ATP in repeated cycles (Basso et al., 2003; Ernst et al., 2008; Gupta et al., 2014; Mishra et al., 2014; Timachi et al., 2017), and 2) alternatively, ATP may be released from the noncanonical NBS in unhydrolyzed form after ATP is hydrolyzed at the canonical NBS. This notion was put forward by Furman et al. (2013) for the yeast full-length transporter Pdr5.

In the present study, we explored interdomain communication paths in BSEP to understand the involvement of the degenerate and the canonical NBS for taurocholate transport. In a first series of experiments, the consequences of conferring hydrolysis competency to NBS1 were explored. The ABCB subfamily is the most diverse of all human subfamilies, containing both half- and full-length transporters, as well as members with one or with two hydrolysis-competent NBSs. Our mutation strategy was thus guided by sequence differences and similarity within the ABCB subfamily. Accordingly, we replaced noncanonical residues in the Walker B motif, the Q-loop, and signature sequence of NBS1 with canonical ones. The noncanonical residues are M584 (Walker B), E502 (Q-loop), and R1221 and E1223 (signature sequence). Replacement of methionine with glutamate in the Walker B motif of NBS1 ensued in the singlemutant M584E. This amino acid change imparted hydrolysis competency to NBS1, as the double-mutant M584E/E1244Q, in 
which mutation of the catalytic glutamate E1244 renders NBS2 hydrolysis incompetent, shows ATPase activity that is comparable to wild-type protein. Thus, the ability of NBS1 to hydrolyze ATP (in the single M584E) does not result in a significant loss of transport activity. Hydrolysis in turn would lead to dissociation of residues in the two nucleotide binding domains, which jointly form NBS1, and thus, a platform function of NBS1 for repeated cycles of hydrolysis in NBS2 is unlikely. The importance of the presence of the three additional noncanonical residues in NBS1 of BSEP presently remains elusive.

In a next series of experiments, we explored the role of selected residues in conserved motifs, namely the Walker A lysines and D-loop aspartates in both NBSs for ATPase activity and taurocholate transport. The Walker A mutants completely lost transport activity, irrespective of the NBS in which they were mutated. These results are in agreement with earlier data on human TAP1/2, in which mutation of conserved lysine residues in the Walker A motifs similarly abolished transport activity (Azzaria et al., 1989; Lapinski et al., 2001). Basal ATPase activity was retained in these mutants, indicating that hydrolytic capacity alone is not sufficient to sustain the transport process.

The D-loops have been indicted to be involved in NBD-NBD communication. The eponymous aspartate is conserved in both NBSs of BSEP. In either of the D-loop mutants, basal ATPase activity was comparable to that of wild-type. Thus, a direct involvement of these residues in ATP hydrolysis seems unlikely. Although the D1250A mutant in NBS1 retained its ability to be stimulated by taurocholate, this property was lost by mutating residue D590 in NBS2. In the latter taurocholate transport was abolished, whereas for analogous mutation in NBS1 (D1250A) transport activity was decreased to 50\%. Similar results were reported for two other ABC transporters with one noncanonical NBS, TM287/288 (Hohl et al., 2014) and Pdr5 (Furman et al., 2013). Taken together, we interpret our data to indicate that the taurocholate binding site is able to communicate the presence of substrate to NBS2, but not to NBS1. For this communication, the Walker A lysine and the D-loop aspartate of NBS2 are required.

In a final set of experiments, we replaced the catalytic glutamate of NBS2 by glutamine. In the ensuing E1244Q mutant, we then introduced a glutamate residue into the Walker B motif of NBS1. This double mutant (M584E/E1244Q) consistently showed $15 \%$ of wild-type transport activity. Time dependencies showed that steady state levels of uptake were $75 \%-80 \%$ reduced in the mutant (Fig. 9), suggesting that this mutant may have lost active and adopted facilitative characteristics. We used a kinetic model to support this notion. This model comprised a term for loss of taurocholate from the vesicle lumen, which we found to occur after washout of taurocholate and ATP from the incubation medium (data not shown). Although this loss did not in any way compromise interpretation of transport data, it was taken advantage of, to corroborate that transport in the mutant was facilitative. The rationale was as follows: loss of taurocholate from the vesicle would only be observed when concentrative transport into the vesicle occurs. This would lead to nonlinear curve characteristics. In contrast,
A
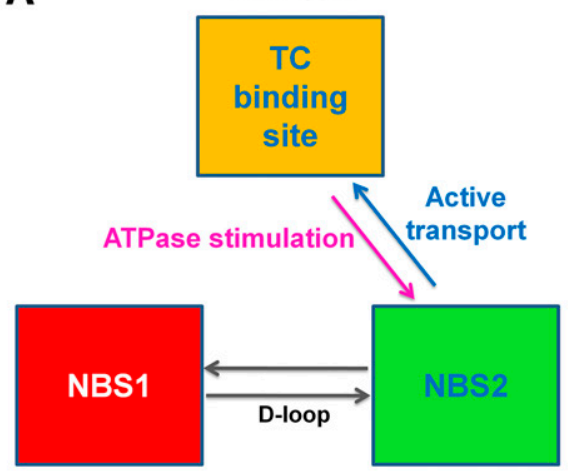

C

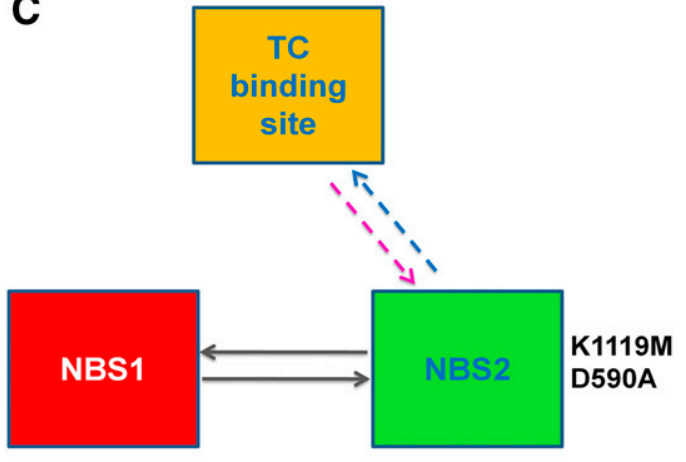

B



D

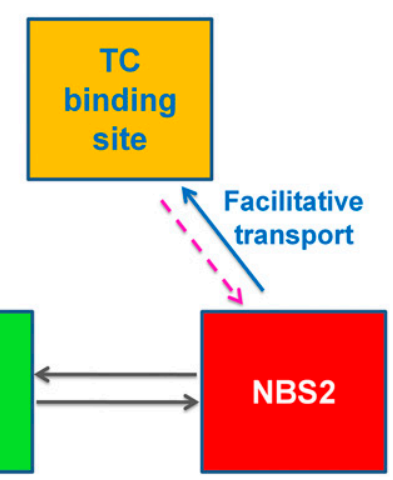

Fig. 11. A model for directional communication between BSEP domains. The taurocholate (TC) binding site (most likely a composite binding site formed by contributions from both TMDs) is highlighted in yellow. The composite ATP binding sites NBS1 and 2 are shown in red (hydrolysis incompetent) and green (hydrolysis competent), respectively. The NBS-NBS communication is indicated by gray arrows. (A) Refers to the situation in wild-type protein. Here the taurocholate binding site signals to NBS2 that substrate has been bound. This is evident from ATPase stimulation experiments, which show that taurocholate stimulates basal ATPase activity (magenta arrow). In contrast, the hydrolysis-competent NBS2 can drive taurocholate transport (blue arrow). The E1244Q mutant (B) is ATPase and transport deficient. Thus, hydrolysis competency of at least one NBS is required for transport. Mutants D590A and K1119M affect NBS2. These mutants (C) are transport deficient. Basal ATPase activity is retained, but stimulation by taurocholate is lost. In the M584E/E1244Q mutant (D), hydrolysis of ATP in NBS1 is considered a prerequisite for discharge of unhydrolyzed ATP from NBS2. This release of ATP resets the transporter into an inwardfacing substrate-binding competent state. 
curves would be expected to be linear for facilitators. This is indeed observed experimentally (Fig. 10). Because of its strict ATP dependence, the M584E/E1244Q mutant has adopted properties of an ATP-gated facilitator. To the best of our knowledge, this has not been shown for any other ABC transporter with one noncanonical NBS. An earlier report by Robert Tampé's group engineered TAP1/2 to act as a facilitator (Grossmann et al., 2014). However, in this case, the change in properties was brought about by mutating the aspartate in the D-loop of TAP1; the transporter differs from the BSEP mutant in that ADP and AMP-PNP can replace ATP in function.

In Fig. 11, we propose a model for the interdomain communication of BSEP based on data from this study. Transport is a measure of the ability of NBS2 (canonical) to communicate conformational changes to the taurocholate binding site. In contrast, stimulation of basal ATPase activity by taurocholate reflects the ability of the taurocholate binding site to signal to NBS2 that substrate has been bound. Both these interdomain communication paths are present in wild-type protein (Fig. 11A), but lost in the E1244Q (Fig. 11B), the K1119M, and the D590A mutant (Fig. 11C). In the M584E/E1244Q mutant (Fig. 11D), taurocholate is transported in facilitative manner. In this study, NBS2 is not able to hydrolyze ATP. However, we propose that ATP is able to dissociate from its binding site in unhydrolyzed form, when ATP is hydrolyzed in NBS1. This ensures that the protein can complete its catalytic cycle to return to a substratebinding competent state. Because of the inability of NBS2 to hydrolyze ATP, substrate affinity would remain higher for the facilitator in the outward-facing conformation and thus result in the loss of active transport characteristics.

With respect to a reduction of the number of hydrolysiscompetent NBSs from two to one in about half of human $\mathrm{ABC}$ transporters (including BSEP and the entire ABCC subfamily), the following can be stated: 1) development of full transporters, from homodimeric half transporters by gene duplication, represented a prerequisite for evolution of these asymmetric proteins (Chen et al., 1986; Ueda et al., 1989; Kerr, 2002). A second path to ABC proteins with only one noncanonical NBS was the evolution of heterodimeric half transporters. This is infrequently observed and only seen for TAP1/2 and ABCG5/G8. 2) A selective advantage may have arisen from the ensuing change in substrate specificity, be it in either broadening it in full transporters with two hydrolysis-competent NBSs or narrowing it in those that only contain one. Any proof of coevolution of residues in the TMDs and causally related changes in NBS1 (evolutionary coupling) (Hopf et al., 2017) should be able to provide evidence for this notion. 3) In BSEP a single hydrolysis-competent NBS may have resulted in restricting substrate specificity to negatively charged bile salts, while excluding compounds of alternative chemistry. Although entirely conjectural at present, the large number of small-molecule drugs, which are capable of direct interaction with (and inhibition of) BSEP, may be a reflection of the presence of an evolutionarily retained former substrate binding site, which has lost coupling to a canonical NBS.

\section{Conclusion}

We engineered BSEP to shed light on the molecular mechanism of taurocholate transport. This allows us to present a model for taurocholate transport with respect to its coupling to the energy-generating step. Our data are expected to form a basis for exploration of the taurocholate binding site and an understanding of the interaction between the transporter and drugs. It is also expected to promote an understanding of the functional biology of other members of the human $\mathrm{ABC}$ protein family that harbor one noncanonical NBS.

\section{Acknowledgments}

The authors are grateful to Bruno Stieger for providing us with plasmids containing the BSEP construct and for continuing support and discussion; Johannes Noe for in-depth discussion of plasmid propagation in Escherichia coli; Enrico Klotzsch for critically reading the manuscript and the Institute of Medical Statistics of the Medical University of Vienna (Florian Frommlet) for expert statistical support.

\section{Authorship Contributions}

Participated in research design: Sohail, Chiba.

Conducted experiments: Sohail, Wlcek.

Contributed new reagents or analytic tools: Schmid.

Performed data analysis: Sohail, Schmid, Spork, Stockner, Chiba. Wrote or contributed to the writing of the manuscript: Sohail, Schmid, Szakács, Trauner, Chiba.

\section{References}

Aller SG, Yu J, Ward A, Weng Y, Chittaboina S, Zhuo R, Harrell PM, Trinh YT, Zhang Q, Urbatsch IL, et al. (2009) Structure of P-glycoprotein reveals a molecular basis for poly-specific drug binding. Science 323:1718-1722.

Al-Shawi MK, Polar MK, Omote H, and Figler RA (2003) Transition state analysis of the coupling of drug transport to ATP hydrolysis by P-glycoprotein. J Biol Chem 278:52629-52640.

Azzaria M, Schurr E, and Gros P (1989) Discrete mutations introduced in the predicted nucleotide-binding sites of the mdr1 gene abolish its ability to confer multidrug resistance. Mol Cell Biol 9:5289-5297.

Bársony O, Szalóki G, Türk D, Tarapcsák S, Gutay-Tóth Z, Bacsó Z, Holb IJ, Székvölgyi L, Szabó G, Csanády L, et al. (2016) A single active catalytic site is sufficient to promote transport in P-glycoprotein. Sci Rep 6:24810.

Basso C, Vergani P, Nairn AC, and Gadsby DC (2003) Prolonged nonhydrolytic interaction of nucleotide with CFTR's NH2-terminal nucleotide binding domain and its role in channel gating. J Gen Physiol 122:333-348.

Bathena SP, Mukherjee S, Olivera M, and Alnouti Y (2013) The profile of bile acids and their sulfate metabolites in human urine and serum. J Chromatogr B Analyt Technol Biomed Life Sci 942-943:53-62.

Chen CJ, Chin JE, Ueda K, Clark DP, Pastan I, Gottesman MM, and Roninson IB (1986) Internal duplication and homology with bacterial transport proteins in the mdr1 (P-glycoprotein) gene from multidrug-resistant human cells. Cell 47:381-389.

Chiba P, Freissmuth M, and Stockner T (2014) Defining the blanks-pharmacochaperoning of SLC6 transporters and ABC transporters. Pharmacol Res 83:63-73.

Chifflet S, Torriglia A, Chiesa R, and Tolosa S (1988) A method for the determination of inorganic phosphate in the presence of labile organic phosphate and high concentrations of protein: application to lens ATPases. Anal Biochem 168:1-4.

Cuperus FJ, Claudel T, Gautherot J, Halilbasic E, and Trauner M (2014) The role of canalicular ABC transporters in cholestasis. Drug Metab Dispos 42:546-560.

Dawson RJ and Locher KP (2006) Structure of a bacterial multidrug ABC transporter. Nature 443:180-185.

Dawson RJ and Locher KP (2007) Structure of the multidrug ABC transporter Sav1866 from Staphylococcus aureus in complex with AMP-PNP. FEBS Lett 581: 935-938.

Dawson S, Stahl S, Paul N, Barber J, and Kenna JG (2012) In vitro inhibition of the bile salt export pump correlates with risk of cholestatic drug-induced liver injury in humans. Drug Metab Dispos 40:130-138.

Dönmez Cakil Y, Khunweeraphong N, Parveen Z, Schmid D, Artaker M, Ecker GF, Sitte HH, Pusch O, Stockner T, and Chiba P (2014) Pore-exposed tyrosine residues of P-glycoprotein are important hydrogen-bonding partners for drugs. Mol Pharmacol 85:420-428.

Ernst R, Kueppers P, Klein CM, Schwarzmueller T, Kuchler K, and Schmitt L (2008) A mutation of the H-loop selectively affects rhodamine transport by the yeast multidrug ABC transporter Pdr5. Proc Natl Acad Sci USA 105:5069-5074.

Furman C, Mehla J, Ananthaswamy N, Arya N, Kulesh B, Kovach I, Ambudkar SV and Golin J (2013) The deviant ATP-binding site of the multidrug efflux pump Pdr5 plays an active role in the transport cycle. J Biol Chem 288:30420-30431.

Gerloff T, Stieger B, Hagenbuch B, Madon J, Landmann L, Roth J, Hofmann AF, and Meier PJ (1998) The sister of P-glycoprotein represents the canalicular bile salt export pump of mammalian liver. J Biol Chem 273:10046-10050.

Grossmann N, Vakkasoglu AS, Hulpke S, Abele R, Gaudet R, and Tampé R (2014) Mechanistic determinants of the directionality and energetics of active export by a heterodimeric ABC transporter. Nat Commun 5:5419.

Gupta RP, Kueppers P, Hanekop N, and Schmitt L (2014) Generating symmetry in the asymmetric ATP-binding cassette (ABC) transporter Pdr5 from Saccharomyces cerevisiae. J Biol Chem 289:15272-15279.

Hartley JL, Temple GF, and Brasch MA (2000) DNA cloning using in vitro sitespecific recombination. Genome Res 10:1788-1795.

Higgins CF (1992) ABC transporters: from microorganisms to man. Annu Rev Cell Biol 8:67-113. 
Hirano M, Maeda K, Hayashi H, Kusuhara H, and Sugiyama Y (2005) Bile salt export pump (BSEP/ABCB11) can transport a nonbile acid substrate, pravastatin. $J$ Pharmacol Exp Ther 314:876-882.

Hohl M, Hürlimann LM, Böhm S, Schöppe J, Grütter MG, Bordignon E, and Seeger MA (2014) Structural basis for allosteric cross-talk between the asymmetric nucleotide binding sites of a heterodimeric ABC exporter. Proc Natl Acad Sci USA 111:11025-11030.

Hopf TA, Ingraham JB, Poelwijk FJ, Schärfe CP, Springer M, Sander C, and Marks DS (2017) Mutation effects predicted from sequence co-variation. Nat Biotechnol 35:128-135.

Hung LW, Wang IX, Nikaido K, Liu PQ, Ames GF, and Kim SH (1998) Crystal structure of the ATP-binding subunit of an ABC transporter. Nature 396:703-707.

Hyde SC, Emsley P, Hartshorn MJ, Mimmack MM, Gileadi U, Pearce SR, Gallagher MP, Gill DR, Hubbard RE, and Higgins CF (1990) Structural model of ATP-binding proteins associated with cystic fibrosis, multidrug resistance and bacterial transport. Nature 346:362-365.

Jin MS, Oldham ML, Zhang Q, and Chen J (2012) Crystal structure of the multidrug transporter P-glycoprotein from Caenorhabditis elegans. Nature 490:566-569.

Kerr ID (2002) Structure and association of ATP-binding cassette transporter nucleotide-binding domains. Biochim Biophys Acta 1561:47-64.

Kubitz R, Dröge C, Stindt J, Weissenberger K, and Häussinger D (2012) The bile salt export pump (BSEP) in health and disease. Clin Res Hepatol Gastroenterol 36:536-553.

Lapinski PE, Neubig RR, and Raghavan M (2001) Walker A lysine mutations of TAP1 and TAP2 interfere with peptide translocation but not peptide binding. $J$ Biol Chem 276:7526-7533.

Mishra S, Verhalen B, Stein RA, Wen PC, Tajkhorshid E, and Mchaourab HS (2014) Conformational dynamics of the nucleotide binding domains and the power stroke of a heterodimeric ABC transporter. eLife 3:e02740.

Montanari F, Pinto M, Khunweeraphong N, Wlcek K, Sohail MI, Noeske T, Boyer S, Chiba P, Stieger B, Kuchler K, et al. (2016) Flagging drugs that inhibit the bile salt export pump. Mol Pharm 13:163-171.

Morgan RE, Trauner M, van Staden CJ, Lee PH, Ramachandran B, Eschenberg M, Afshari CA, Qualls CW, Jr, Lightfoot-Dunn R, and Hamadeh HK (2010) Interference with bile salt export pump function is a susceptibility factor for human liver injury in drug development. Toxicol Sci 118:485-500.

Müller M, Meijer C, Zaman GJ, Borst P, Scheper RJ, Mulder NH, de Vries EG, and Jansen PL (1994) Overexpression of the gene encoding the multidrug resistance-associated protein results in increased ATP-dependent glutathione S-conjugate transport. Proc Natl Acad Sci USA 91:13033-13037.

Parveen Z, Stockner T, Bentele C, Pferschy S, Kraupp M, Freissmuth M, Ecker GF, and Chiba P (2011) Molecular dissection of dual pseudosymmetric solute translocation pathways in human P-glycoprotein. Mol Pharmacol 79:443-452.

Pedersen JM, Matsson P, Bergström CA, Hoogstraate J, Norén A, LeCluyse EL, and Artursson P (2013) Early identification of clinically relevant drug interactions with the human bile salt export pump (BSEP/ABCB11). Toxicol Sci 136:328-343.

Procko E, O'Mara ML, Bennett WF, Tieleman DP, and Gaudet R (2009) The mechanism of $\mathrm{ABC}$ transporters: general lessons from structural and functional studies of an antigenic peptide transporter. FASEB J 23:1287-1302.
Ritschel T, Hermans SM, Schreurs M, van den Heuvel JJ, Koenderink JB, Greupink $\mathrm{R}$, and Russel FG (2014) In silico identification and in vitro validation of potential cholestatic compounds through 3D ligand-based pharmacophore modeling of BSEP inhibitors. Chem Res Toxicol 27:873-881.

Sali A and Blundell TL (1993) Comparative protein modelling by satisfaction of spatial restraints. J Mol Biol 234:779-815.

Schmid D, Ecker G, Kopp S, Hitzler M, and Chiba P (1999) Structure-activity relationship studies of propafenone analogs based on P-glycoprotein ATPase activity measurements. Biochem Pharmacol 58:1447-1456.

Schmid D, Koenig X, Bulusu S, Schicker K, Freissmuth M, Sitte HH, and Sandtner W (2015) The conservative view: is it necessary to implant a stent into the dopamine transporter? Br J Pharmacol 172:4775-4778.

Schmidt H and Jirstrand M (2006) Systems biology toolbox for MATLAB: a computational platform for research in systems biology. Bioinformatics 22 514-515.

Shintre CA, Pike AC, Li Q, Kim JI, Barr AJ, Goubin S, Shrestha L, Yang J, Berridge G, Ross J, et al. (2013) Structures of ABCB10, a human ATP-binding cassette transporter in apo- and nucleotide-bound states. Proc Natl Acad Sci USA 110 9710-9715.

Spork M, Sohail MI, Schmid D, Ecker GF, Freissmuth M, Chiba P, and Stockner T (2017) Folding correction of ABC-transporter ABCB1 by pharmacological chaperones: a mechanistic concept. Pharmacol Res Perspect 5:e00325.

Stieger B, Meier Y, and Meier PJ (2007) The bile salt export pump. Pflugers Arch 453:611-620.

Stockner T, de Vries SJ, Bonvin AM, Ecker GF, and Chiba P (2009) Data-driven homology modelling of P-glycoprotein in the ATP-bound state indicates flexibility of the transmembrane domains. FEBS $J$ 276:964-972.

Timachi MH, Hutter CA, Hohl M, Assafa T, Böhm S, Mittal A, Seeger MA and Bordignon E (2017) Exploring conformational equilibria of a heterodimeric ABC transporter. eLife 6 .

Ueda K, Yamano Y, Kioka N, Kakehi Y, Yoshida O, Gottesman MM, Pastan I, and Komano T (1989) Detection of multidrug resistance (MDR1) gene RNA expression in human tumors by a sensitive ribonuclease protection assay. Jpn $J$ Cancer Res 80:1127-1132.

Walker JE, Saraste M, Runswick MJ, and Gay NJ (1982) Distantly related sequences in the alpha- and beta-subunits of ATP synthase, myosin, kinases and other ATP-requiring enzymes and a common nucleotide binding fold. EMBO $J$ 1: 945-951.

Wlcek K, Hofstetter L, and Stieger B (2014) Transport of estradiol-17ß-glucuronide, estrone-3-sulfate and taurocholate across the endoplasmic reticulum membrane: evidence for different transport systems. Biochem Pharmacol 88:106-118.

Address correspondence to: Peter Chiba, Institute of Medical Chemistry, Center for Pathobiochemistry and Genetics, Medical University of Vienna, Waehringerstrasse 10, A-1090 Vienna, Austria. E-mail: peter.chiba@meduniwien. ac at 